\title{
ASYMPTOTICAL PROPERTIES OF DISTRIBUTIONS OF ISOTROPIC LÉVY PROCESSES
}

\author{
PANKI KIM AND ANTE MIMICA $(\dagger)$
}

\begin{abstract}
In this paper, we establish the precise asymptotic behaviors of the tail probability and the transition density of a large class of isotropic Lévy processes when the scaling order is between 0 and 2 including 2. We also obtain the precise asymptotic behaviors of the tail probability of subordinators when the scaling order is between 0 and 1 including 1 .

The asymptotic expressions are given in terms of the radial part of characteristic exponent $\psi$ and its derivative. In particular, when $\psi(\lambda)-\frac{\lambda}{2} \psi^{\prime}(\lambda)$ varies regularly, as $\frac{t \psi\left(r^{-1}\right)^{2}}{\psi\left(r^{-1}\right)-(2 r)^{-1} \psi^{\prime}\left(r^{-1}\right)} \rightarrow 0$ the tail probability $\mathbb{P}\left(\left|X_{t}\right| \geq r\right)$ is asymptotically equal to a constant times $t\left(\psi\left(r^{-1}\right)-(2 r)^{-1} \psi^{\prime}\left(r^{-1}\right)\right)$.
\end{abstract}

\section{Introduction AND MAin RESUlts}

This paper is a continuation of the journey of the second-named author on investigating the behavior of the transition density of Lévy processes whose (weak) scaling order is between 0 and 2 including 2. In [Mim], the second-named author considered the large class of purely discontinuous subordinate Brownian motions when the weak scaling order is between 0 and 2 including 2 , and obtained sharp heat kernel estimates of such processes. In this paper, we assume that the function $\psi(\lambda)-\frac{\lambda}{2} \psi^{\prime}(\lambda)$ is regularly varying where $\xi \rightarrow \psi(|\xi|)$ is the characteristic exponent of an isotropic Lévy process, and discuss the asymptotic behavior of the tail probability. As a corollary, when the process is unimodal, the asymptotic behavior of the tail probability implies the precise off-diagonal asymptotic expression of the transition density. We remark here that the function $\psi(\lambda)-\frac{\lambda}{2} \psi^{\prime}(\lambda)$ is a natural counterpart of the function $H(\lambda)=\phi(\lambda)-\lambda \phi^{\prime}(\lambda)$ for subordinator with Laplace exponent $\phi$, which has been already known in the literature (see [JP87]).

2010 Mathematics Subject Classification. Primary 60G51, 60J35, Secondary 60F99.

Key words and phrases. Asymptotic, transition density, Lévy process, unimodal Lévy process, heat kernel, Laplace exponent, Lévy measure, subordinator, subordinate Brownian motion.

This work was supported by the National Research Foundation of Korea(NRF) grant funded by the Korea government(MSIP) (No. 2016R1E1A1A01941893). 
Lévy processes and their associated (non-local) operators have been of current research interest both in probability theory and in PDE. The transition density of a Lévy process $X$ is also the fundamental solution of corresponding non-local operator. Except a few special case, the explicit expression of transition density of Lévy process is typically impossible to get. Thus obtaining the exact off-diagonal asymptotic expression of transition density is an important problem both in probability theory and in analysis.

Even though transition densities of Lévy processes are determined by their characteristic exponents, in general, neither the transition density nor the tail probability can be calculated explicitly by their characteristic exponents. But for large class of isotropic Lévy process, their asymptotic behaviors can be formulated in terms of their characteristic exponents. For example, for the transition density $p(t, x)$ of isotropic $\alpha$-stable process, whose characteristic exponent is $|x|^{\alpha}$, it is known that

$$
\lim _{t|x|^{-\alpha} \rightarrow 0} \frac{p(t, x)}{t|x|^{-d-\alpha}}=\alpha 2^{\alpha-1} \pi^{-d / 2-1} \sin \left(\frac{\alpha \pi}{2}\right) \Gamma\left(\frac{\alpha}{2}\right) \Gamma\left(\frac{d+\alpha}{2}\right),
$$

where $\Gamma(t)$ is the gamma function. (See [BG60].) Since, in general, the Lévy kernel of isotropic Lévy process $X$ is not equal to a constant times $|x|^{-d} \psi\left(|x|^{-1}\right)$ where $\xi \rightarrow \psi(|\xi|)$ is the characteristic exponent of $X$, we do not expect that (1.1) holds. But as it is shown in [CGT15, Theorems 4 and 5], when the characteristic exponent $\psi$ of isotropic unimodal Lévy process is regularly varying at $\infty$ (at zero, respectively) with index $\alpha \in(0,2)$, the transition density is asymptotically equal to a constant times $t|x|^{-d} \psi\left(|x|^{-1}\right)$ as $t \psi\left(|x|^{-1}\right) \rightarrow 0$ and $|x| \rightarrow \infty$ (|x| $\rightarrow 0$, respectively).

Natural open questions are, when the regularly varying index $\alpha$ is not strictly between 0 and 2 ,

(1) instead of $t \psi\left(|x|^{-1}\right) \rightarrow 0$, what condition in the limit we need to put?

(2) to what functions the tail probability and the transition density, respectively, are asymptotically equal?

In this paper, we answer the above questions when $\psi(\lambda)-\frac{\lambda}{2} \psi^{\prime}(\lambda)$ varies regularly. Up to our knowledge, the asymptotic expressions in Theorems 1.1, 1.3(ii) and 1.4(ii) are new forms comparing to existing results (for example, [Ben94, BG60, CGT15, FLH09, Ish94, Kno11, Léa87]) and it also covers [CGT15, Theorems 4 and 5], see Theorems 1.3(i) and 1.4(i).

In this paper, $f(t) \sim g(t), t \rightarrow a$ means $\lim _{t \rightarrow a} f(t) / g(t)=1$. We also use the notation $f(t, r) \sim g(t, r), r \rightarrow a$ and $h(t, r) \rightarrow 0$, which means

$$
\lim _{\substack{r \rightarrow a \\ h(t, r) \rightarrow 0}} \frac{f(t, r)}{g(t, r)}=1 .
$$


We say a function $\ell:(0, \infty) \rightarrow(0, \infty)$ varies regularly at $\infty$ (at zero, respectively) with index $\alpha$ if $\ell(\lambda t) / \ell(t) \rightarrow \lambda^{\alpha}$ as $t \rightarrow \infty$ ( $t \rightarrow 0$, respectively) for every $\lambda>0$.

$T=\left(T_{t}\right)_{t \geq 0}$ is called a subordinator if it is a non-decreasing Lévy process. The Laplace transform of the law of a subordinator $T_{t}$ is given by

$$
\mathbb{E} e^{-\lambda T_{t}}=e^{-t \phi(\lambda)}, \quad \lambda>0,
$$

and $\phi:(0, \infty) \rightarrow(0, \infty)$ is called the Laplace exponent of the subordinator $T$.

The first main result of this paper is the following asymptotic expression of the tail probability of a subordinator.

Theorem 1.1. Suppose that $\phi$ is the Laplace exponent of a subordinator $T$. If $H(\lambda)=\phi(\lambda)-\lambda \phi^{\prime}(\lambda)$ varies regularly at 0 (at $\infty$, respectively) with index $\gamma \in[0,2)$, then

$$
\mathbb{P}\left(T_{t} \geq r\right) \sim \frac{1}{\Gamma(2-\gamma)} t H\left(r^{-1}\right), \quad r \rightarrow \infty(r \rightarrow 0, \text { resp. }) \quad \text { and } t \frac{\phi\left(r^{-1}\right)^{2}}{H\left(r^{-1}\right)} \rightarrow 0
$$

It turns out that, in fact, the tail of the Lévy measure of $T$ describes decay of $\mathbb{P}\left(T_{t} \geq r\right)$. (See Proposition 2.3 below.)

Note that, if $\phi$ varies regularly at 0 (at $\infty$, respectively) with index $\gamma \in(0,1)$, since $\phi^{\prime}$ is non-increasing, we can apply monotone density theorem (see [BGT87, Theorem 1.7.2]) to get $\phi^{\prime}(\lambda) \sim \gamma \lambda^{-1} \phi(\lambda)$ as $\lambda \rightarrow 0$. Hence,

$$
\lim _{\substack{\lambda \rightarrow 0 \\(\lambda \rightarrow \infty, \text { resp. })}} \frac{H(\lambda)}{\phi(\lambda)}=1-\lim _{\substack{\lambda \rightarrow 0 \\(\lambda \rightarrow \infty, \text { resp. })}} \frac{\lambda \phi^{\prime}(\lambda)}{\phi(\lambda)}=1-\gamma .
$$

(1.4) and Proposition 3.7 below imply that if $\gamma \in(0,1)$ then $\phi$ varies regularly at 0 with index $\gamma$ if and only if $H$ varies regularly at 0 with index $\gamma$. Furthermore, from (1.4) we see that for $\gamma=1$ functions $H$ and $\phi$ are not comparable, but we will see that regular variation of $H$ implies regular variation of $\phi$ (see Proposition 3.7 below).

A consequence of Theorem 1.1 and (1.4) is that, if $\gamma \in[0,1)$, we may replace condition $t \frac{\phi\left(r^{-1}\right)^{2}}{H\left(r^{-1}\right)} \rightarrow 0$ in $(1.3)$ by $t \phi\left(r^{-1}\right) \rightarrow 0$.

Corollary 1.2. Assume that $\phi$ varies regularly at 0 (at $\infty$, respectively) with index $\gamma \in[0,1)$. Then

$$
\mathbb{P}\left(T_{t} \geq r\right) \sim \frac{1}{\Gamma(1-\gamma)} t \phi\left(r^{-1}\right), \quad r \rightarrow \infty(r \rightarrow 0, \text { resp. }) \quad \text { and } \quad t \phi\left(r^{-1}\right) \rightarrow 0 .
$$

The next main result is the precise asymptotic expression of the tail probability of isotropic Lévy processes. We recall that $\xi \rightarrow \psi(|\xi|)$ is the characteristic exponent of 
isotropic Lévy process $X$ if

$$
\mathbb{E}\left[e^{i \xi \cdot X_{t}}\right]=e^{-t \psi(|\xi|)}, \quad \xi \in \mathbb{R}^{d}
$$

where

$$
\psi(|\xi|)=a|\xi|^{2}+\int_{\mathbb{R}^{d}}\left(1-e^{i \xi \cdot x}\right) \nu(d x)
$$

with $a \geq 0$ and a Lévy measure $\nu$.

Theorem 1.3. Suppose $X=\left(X_{t}\right)_{t \geq 0}$ is an isotropic Lévy process in $\mathbb{R}^{d}, d \geq 1$ with the characteristic exponent $\xi \rightarrow \psi(|\xi|)$. Let $g(\lambda)=\psi(\lambda)-\frac{\lambda}{2} \psi^{\prime}(\lambda)$.

(i) Suppose that $g$ varies regularly at 0 with index $\alpha \in[0,4)$. Then

$$
\begin{aligned}
& \mathbb{P}\left(\left|X_{t}\right| \geq r\right) \sim \frac{2^{\alpha-1} \Gamma\left(\frac{d+\alpha}{2}\right)}{\Gamma\left(2-\frac{\alpha}{2}\right) \Gamma\left(\frac{d}{2}\right)} \operatorname{tg}\left(r^{-1}\right), \quad r \rightarrow \infty \\
& \quad \text { and } \frac{t \psi\left(r^{-1}\right)^{2}}{\psi\left(r^{-1}\right)-(2 r)^{-1} \psi^{\prime}\left(r^{-1}\right)}=\frac{t \psi\left(r^{-1}\right)}{1-\frac{r^{-1} \psi^{\prime}\left(r^{-1}\right)}{2 \psi\left(r^{-1}\right)}} \rightarrow 0 .
\end{aligned}
$$

(ii) Suppose that $g$ varies regularly at $\infty$ with index $\alpha \geq 0$. Then, in fact, $g$ varies regularly at $\infty$ with index $\alpha \in[0,2]$. We further assume that $\psi$ does not have diffusion part ( $a=0$ in (1.5)) if $g$ varies regularly at $\infty$ with index $\alpha \in[0,2)$. Then

$$
\mathbb{P}\left(\left|X_{t}\right| \geq r\right) \sim \frac{2^{\alpha-1} \Gamma\left(\frac{d+\alpha}{2}\right)}{\Gamma\left(2-\frac{\alpha}{2}\right) \Gamma\left(\frac{d}{2}\right)} \operatorname{tg}\left(r^{-1}\right), \quad r \rightarrow 0 \text { and } \frac{t \psi\left(r^{-1}\right)^{2}}{\psi\left(r^{-1}\right)-(2 r)^{-1} \psi^{\prime}\left(r^{-1}\right)} \rightarrow 0 .
$$

Finally we give the precise off-diagonal asymptotic expression of the transition density $p(t, x)$ when the isotropic Lévy process is unimodal, that is, $r \rightarrow q(t, r)$ is decreasing where $p(t, x)=q(t,|x|)$.

Theorem 1.4. Suppose $X=\left(X_{t}\right)_{t \geq 0}$ is a unimodal Lévy process in $\mathbb{R}^{d}, d \geq 1$ with the characteristic exponent $\xi \rightarrow \psi(|\xi|)$, and that $p(t, x)$ and $J(x)$ are the transition density and the Lévy kernel of $X$, respectively. Suppose that $g(\lambda)=\psi(\lambda)-\frac{\lambda}{2} \psi^{\prime}(\lambda)$ varies regularly at 0 with index $\alpha \in(0,4)$ (at $\infty$ with index $\alpha \in(0,2]$, respectively). We further assume that $\psi$ does not have diffusion part $(a=0$ in (1.5)) if $g$ varies regularly at $\infty$ with index $\alpha \in(0,2)$. Then

$$
\begin{aligned}
p(t, x) \sim \alpha 2^{\alpha-1} \pi^{-d / 2} \frac{\Gamma\left(\frac{d+\alpha}{2}\right)}{\Gamma\left(2-\frac{\alpha}{2}\right)} t|x|^{-d} g\left(|x|^{-1}\right), \\
\quad|x| \rightarrow \infty(|x| \rightarrow 0, \text { resp. }) \quad \text { and } \quad \frac{t \psi\left(|x|^{-1}\right)}{1-\frac{|x|^{-1} \psi^{\prime}\left(|x|^{-1}\right)}{2 \psi\left(|x|^{-1}\right)}} \rightarrow 0,
\end{aligned}
$$


and

$$
J(x) \sim \alpha 2^{\alpha-1} \pi^{-d / 2} \frac{\Gamma\left(\frac{d+\alpha}{2}\right)}{\Gamma\left(2-\frac{\alpha}{2}\right)}|x|^{-d} g\left(|x|^{-1}\right), \quad|x| \rightarrow \infty(|x| \rightarrow 0, \text { resp. }) .
$$

We conclude this introduction by setting up some notation and conventions. We use ":=" to denote a definition, which is read as "is defined to be"; we denote $a \wedge b:=\min \{a, b\}, a \vee b:=\max \{a, b\}$.

\section{TAIL ASYMPTOTIC BEHAVIORS OF SUBORDINATORS}

In this section we always assume that $T=\left(T_{t}\right)_{t \geq 0}$ is a subordinator and that $\phi$ : $(0, \infty) \rightarrow(0, \infty)$ is the Laplace exponent of $T$. $\phi$ has the following form (see [Ben94, Section 3])

$$
\phi(\lambda)=b \lambda+\int_{(0, \infty)}\left(1-e^{-\lambda t}\right) \mu(d t) .
$$

Here $b \geq 0$ is called the drift and $\mu$ is the Lévy measure of the subordinator $T$, i.e. a measure on $(0, \infty)$ satisfying $\int_{(0, \infty)}(1 \wedge t) \mu(d t)<\infty$. Laplace exponent $\phi$ belongs to the class of Bernstein functions, i.e., a non-negative $C^{\infty}(0, \infty)$ function such that $(-1)^{n} \phi^{(n)} \leq 0$ for all $n \in \mathbb{N}$.

Let us define the function $H:(0, \infty) \rightarrow[0, \infty)$ by

$$
H(\lambda)=\phi(\lambda)-\lambda \phi^{\prime}(\lambda), \quad \lambda>0
$$

Note that, by the concavity of $\phi, H(\lambda) \geq 0$. Moreover, $H$ is non-decreasing since $H^{\prime}(\lambda)=-\lambda \phi^{\prime \prime}(\lambda) \geq 0$. We also note that

$$
H(\lambda)=-\lambda^{2}\left(\frac{\phi(\lambda)}{\lambda}\right)^{\prime}
$$

We remark here that $H$ loses the information on the drift of $\phi$.

In this section we obtain asymptotical properties of the function $\mathbb{P}\left(T_{t} \geq r\right)$ as $r \rightarrow \infty$ $\left(r \rightarrow 0\right.$, respectively) and $t \frac{\phi\left(r^{-1}\right)^{2}}{H\left(r^{-1}\right)} \rightarrow 0$. Note, in particular, that in this case $t \phi\left(r^{-1}\right) \rightarrow 0$, since $H(\lambda) \leq \phi(\lambda)$ for all $\lambda>0$.

Using (1.2) and Fubini theorem, we obtain

$$
\int_{0}^{\infty} e^{-\lambda r} \mathbb{P}\left(T_{t} \geq r\right) d r=\mathbb{E} \int_{0}^{T_{t}} e^{-\lambda r} d r=\mathbb{E}\left[\frac{1-e^{-\lambda T_{t}}}{\lambda}\right]=\frac{1-e^{-t \phi(\lambda)}}{\lambda} .
$$

For a measure $\nu$ on $[0, \infty)$ its Laplace transform $\mathcal{L} \nu$ is defined by

$$
(\mathcal{L} \nu)(\lambda)=\int_{[0, \infty)} e^{-\lambda y} \nu(d y), \quad \lambda>0
$$


In particular, the Laplace transform of the measure $\nu(d y)=\mathbb{P}\left(T_{t} \geq y\right) d y$ is, by (2.4), given by $(\mathcal{L} \nu)(\lambda)=\frac{1-e^{-t \phi(\lambda)}}{\lambda}$ for $\lambda>0$.

We will use the following result which is a part of the continuity theorem for Laplace transforms (see [Fel71, Theorem XIII.1.2] for a proof).

Theorem 2.1. Let $\left(\nu_{n}\right)_{n \in \mathbb{N}}$ be a sequence of measures on $[0, \infty)$ and $\lambda_{0} \geq 0$ such that $\Phi(\lambda):=\lim _{n \rightarrow \infty}\left(\mathcal{L} \nu_{n}\right)(\lambda)$ exists for all $\lambda>\lambda_{0}$. Then $\Phi$ is the Laplace transform of a measure $\nu$ on $[0, \infty)$ and the following convergence holds

$$
\lim _{n \rightarrow \infty} \nu_{n}([0, x])=\nu([0, x])
$$

for all $x \geq 0$ such that $y \mapsto \nu([0, y])$ is continuous at $y$.

Now we prove the main result of this section, namely the tail estimate of the subordinator.

Proof of Theorem 1.1. Taking derivative in (2.4) we obtain

$$
\int_{0}^{\infty} e^{-\lambda r} r \mathbb{P}\left(T_{t} \geq r\right) d r=\frac{1-e^{-t \phi(\lambda)}-t \lambda \phi^{\prime}(\lambda) e^{-t \phi(\lambda)}}{\lambda^{2}}, \quad \lambda>0 .
$$

Using

$$
\begin{aligned}
& 1-e^{-t \phi\left(\lambda x^{-1}\right)}-t \lambda x^{-1} \phi^{\prime}\left(\lambda x^{-1}\right) e^{-t \phi\left(\lambda x^{-1}\right)} \\
= & 1-e^{-t \phi\left(\lambda x^{-1}\right)}-t \phi\left(\lambda x^{-1}\right) e^{-t \phi\left(\lambda x^{-1}\right)}+t H\left(\lambda x^{-1}\right) e^{-t \phi\left(\lambda x^{-1}\right)}
\end{aligned}
$$

and

$$
1-e^{-t \phi\left(\lambda x^{-1}\right)}-t \phi\left(\lambda x^{-1}\right) e^{-t \phi\left(\lambda x^{-1}\right)} \leq \frac{1}{2}\left(t \phi\left(\lambda x^{-1}\right)\right)^{2}
$$

in $(2.5)$ it follows that

$$
\begin{aligned}
& \frac{\int_{0}^{\infty} e^{-\lambda x^{-1} r} r \mathbb{P}\left(T_{t} \geq r\right) d r}{t x^{2} H\left(\lambda x^{-1}\right)}=\frac{1-e^{-t \phi\left(\lambda x^{-1}\right)}-t \lambda x^{-1} \phi^{\prime}\left(\lambda x^{-1}\right) e^{-t \phi\left(\lambda x^{-1}\right)}}{\lambda^{2} t H\left(\lambda x^{-1}\right)} \\
= & \frac{1-e^{-t \phi\left(\lambda x^{-1}\right)}-t \phi\left(\lambda x^{-1}\right) e^{-t \phi\left(\lambda x^{-1}\right)}}{\lambda^{2} t H\left(\lambda x^{-1}\right)}+\lambda^{-2} e^{-t \phi\left(\lambda x^{-1}\right)} \\
\leq & \frac{t \phi\left(\lambda x^{-1}\right)^{2}}{2 \lambda^{2} H\left(\lambda x^{-1}\right)}+\lambda^{-2} e^{-t \phi\left(\lambda x^{-1}\right)}, \quad \lambda, x>0 .
\end{aligned}
$$

Note that, by using $\phi(\lambda y) \leq(1 \vee y) \phi(\lambda)$ and [Mim, Lemma 2.1],

$$
0 \leq t \frac{\phi\left(\lambda x^{-1}\right)^{2}}{H\left(\lambda x^{-1}\right)}=t \frac{\phi\left(x^{-1}\right)^{2}}{H\left(x^{-1}\right)}\left(\frac{\phi\left(\lambda x^{-1}\right)}{\phi\left(x^{-1}\right)}\right)^{2} \frac{H\left(x^{-1}\right)}{H\left(\lambda x^{-1}\right)} \leq t \frac{\phi\left(x^{-1}\right)^{2}}{H\left(x^{-1}\right)}\left(1 \vee \lambda^{2}\right)\left(1 \vee \lambda^{-2}\right)
$$


Thus $t \frac{\phi\left(x^{-1}\right)^{2}}{H\left(x^{-1}\right)} \rightarrow 0$ implies that $t \frac{\phi\left(\lambda x^{-1}\right)^{2}}{H\left(\lambda x^{-1}\right)} \rightarrow 0$ for any $\lambda>0$ (and so $t \phi\left(\lambda x^{-1}\right) \rightarrow 0$ for any $\lambda>0)$. Therefore from (2.6) we see that

$$
\lim _{t \frac{\phi\left(x^{-1}\right)^{2}}{H\left(x^{-1}\right)} \rightarrow 0} \frac{\int_{0}^{\infty} e^{-\lambda x^{-1} r} r \mathbb{P}\left(T_{t} \geq r\right) d r}{t x^{2} H\left(\lambda x^{-1}\right)}=\lambda^{-2} \quad \text { for all } \quad \lambda>0 .
$$

We now assume that $H(\lambda)=\phi(\lambda)-\lambda \phi^{\prime}(\lambda)$ varies regularly at 0 (at $\infty$, respectively) with index $\gamma \in[0,2)$. Then, changing variables in the integral of the last display and using regular variation of $H$, we conclude that for any $\lambda>0$ the following holds

$$
\begin{aligned}
\lambda^{-2} & =\lim _{\substack{x \rightarrow \infty(x \rightarrow 0, \text { resp. }) \\
t \frac{\phi\left(x^{-1}\right)^{2} \rightarrow 0}{H\left(x^{-1}\right)} \rightarrow 0}} \frac{\int_{0}^{\infty} e^{-\lambda r} x r \mathbb{P}\left(T_{t} \geq x r\right) x d r}{t x^{2} H\left(x^{-1}\right)} \cdot \frac{H\left(x^{-1}\right)}{H\left(\lambda x^{-1}\right)} \\
& =\lim _{\substack{x \rightarrow \infty(x \rightarrow 0, \text { resp. }) \\
t \frac{\phi\left(x^{-1}\right)^{2}}{H\left(x^{-1}\right)} \rightarrow 0}} \frac{\int_{0}^{\infty} e^{-\lambda r} r \mathbb{P}\left(T_{t} \geq x r\right) d r}{t H\left(x^{-1}\right)} \lambda^{-\gamma} .
\end{aligned}
$$

We rewrite the last display in the following way;

$$
\lim _{\substack{x \rightarrow \infty(x \rightarrow 0, \text { resp. }) \\ t \frac{\phi\left(x^{-1}\right)^{2}}{H\left(x^{-1}\right)} \rightarrow 0}} \frac{\int_{0}^{\infty} e^{-\lambda r} r \mathbb{P}\left(T_{t} \geq x r\right) d r}{t H\left(x^{-1}\right)}=\lambda^{\gamma-2}=\int_{0}^{\infty} e^{-\lambda r} \frac{r^{1-\gamma}}{\Gamma(2-\gamma)} d r .
$$

Hence, by using Theorem 2.1 we obtain that for all $R>0$

$$
\lim _{\substack{x \rightarrow \infty(x \rightarrow 0, \text { resp. }) \\ t \frac{\phi\left(x^{-1}\right)^{2} \rightarrow 0}{H\left(x^{-1}\right)} \rightarrow 0}} \frac{\int_{0}^{R} r \mathbb{P}\left(T_{t} \geq x r\right) d r}{t H\left(x^{-1}\right)}=\int_{0}^{R} \frac{r^{1-\gamma}}{\Gamma(2-\gamma)} d r=\frac{R^{2-\gamma}}{\Gamma(3-\gamma)} .
$$

In particular, for any $0<a<1$ we have

$$
\frac{1-a^{2-\gamma}}{\Gamma(3-\gamma)}=\lim _{\substack{x \rightarrow \infty(x \rightarrow 0, \text { resp. }) \\ t \frac{\phi\left(x^{-1}\right)^{2}}{H\left(x^{-1}\right)} \rightarrow 0}} \frac{\int_{a}^{1} r \mathbb{P}\left(T_{t} \geq x r\right) d r}{t H\left(x^{-1}\right)} \geq \limsup _{\substack{x \rightarrow \infty(x \rightarrow 0, \text { resp. }) \\ t \frac{\phi\left(x^{-1}\right)^{2}}{H\left(x^{-1}\right)} \rightarrow 0}} \frac{\left(1-a^{2}\right) \mathbb{P}\left(T_{t} \geq x\right)}{2 t H\left(x^{-1}\right)}
$$

i.e.,

$$
\limsup _{\substack{x \rightarrow \infty\left(x \rightarrow 0, \text { resp. } \\ t \frac{\phi\left(x^{-1}\right)}{H\left(x^{-1}\right)} \rightarrow 0\right.}} \frac{\mathbb{P}\left(T_{t} \geq x\right)}{t H\left(x^{-1}\right)} \leq \frac{2}{\Gamma(3-\gamma)} \frac{1-a^{2-\gamma}}{1-a^{2}} .
$$

Letting $a$ go to 1 we obtain

$$
\limsup _{\substack{x \rightarrow \infty(x \rightarrow 0, \text { resp. }) \\ t \frac{\phi\left(x^{-1}\right)^{2}}{H\left(x^{-1}\right)} \rightarrow 0}} \frac{\mathbb{P}\left(T_{t} \geq x\right)}{t H\left(x^{-1}\right)} \leq \frac{2-\gamma}{\Gamma(3-\gamma)}=\frac{1}{\Gamma(2-\gamma)} .
$$


Similarly, for $b>1$ we obtain

$$
\frac{b^{2-\gamma}-1}{\Gamma(3-\gamma)}=\lim _{\substack{x \rightarrow \infty(x \rightarrow 0, \text { resp. }) \\ t \frac{\phi\left(x^{-1}\right)^{2}}{H\left(x^{-1}\right)} \rightarrow 0}} \frac{\int_{1}^{b} r \mathbb{P}\left(T_{t} \geq x r\right) d r}{t H\left(x^{-1}\right)} \leq \liminf _{\substack{x \rightarrow \infty(x \rightarrow 0, \text { resp. }) \\ t \frac{\phi\left(x^{-1}\right)^{2}}{H\left(x^{-1}\right)} \rightarrow 0}} \frac{\left(b^{2}-1\right) \mathbb{P}\left(T_{t} \geq x\right)}{2 t H\left(x^{-1}\right)}
$$

and, by letting $b$ go to 1 , we obtain

$$
\liminf _{\substack{x \rightarrow \infty(x \rightarrow 0, \text { resp. }) \\ t \frac{\phi\left(x^{-1}\right)^{2}}{H\left(x^{-1}\right)} \rightarrow 0}} \frac{\mathbb{P}\left(T_{t} \geq x\right)}{t H\left(x^{-1}\right)} \geq \frac{1}{\Gamma(2-\gamma)}
$$

The result follows now from (2.8) and (2.9).

Remark 2.2. It is instructive to see why previous proof does not always work if we start with (2.4) instead of (2.5). Assume that $\phi$ varies regularly at 0 with index $\gamma=1$. Using the same idea as in the proof of Theorem 2.1 we obtain

$$
\lim _{\substack{x \rightarrow \infty \\ t \phi\left(x^{-1}\right) \rightarrow 0}} \frac{\int_{0}^{\infty} e^{-\lambda r} \mathbb{P}\left(T_{t} \geq x r\right) d r}{t \phi\left(x^{-1}\right)}=\lambda^{\gamma-1}=1=\int_{0}^{\infty} e^{-\lambda r} \delta_{0}(d r),
$$

where $\delta_{0}$ is the point mass at 0 , i.e.

$$
\delta_{0}(A)= \begin{cases}1, & 0 \in A \\ 0, & 0 \notin A\end{cases}
$$

By continuity theorem, Theorem 2.1, we obtain

$$
\lim _{\substack{x \rightarrow \infty \\ t \phi\left(x^{-1}\right) \rightarrow 0}} \frac{\int_{0}^{R} \mathbb{P}\left(T_{t} \geq x r\right) d r}{t \phi\left(x^{-1}\right)}=\int_{0}^{R} \delta_{0}(d r)=1
$$

implying

$$
\limsup _{\substack{x \rightarrow \infty \\ t \phi\left(x^{-1}\right) \rightarrow 0}} \frac{\mathbb{P}\left(T_{t} \geq x\right)}{2 t \phi\left(x^{-1}\right)} \leq \lim _{\substack{x \rightarrow \infty \\ t \phi\left(x^{-1}\right) \rightarrow 0}} \frac{\int_{1 / 2}^{1} \mathbb{P}\left(T_{t} \geq x r\right) d r}{t \phi\left(x^{-1}\right)}=1-1=0 .
$$

Hence, in this case tail decays faster than $t \phi\left(x^{-1}\right)$ as $x \rightarrow \infty$ and $t \phi\left(x^{-1}\right) \rightarrow 0$.

Proof of Corollary 1.2. Since $1-\gamma>0$, by (1.4), it follows that $H$ varies regularly at 0 (at $\infty$, respectively) with index $\gamma$. Furthermore,

$$
t \frac{\phi\left(r^{-1}\right)^{2}}{H\left(r^{-1}\right)} \rightarrow 0 \quad \text { if and only if } \quad t \phi\left(r^{-1}\right) \rightarrow 0 \quad \text { when } \quad r \rightarrow \infty
$$


and so, by Theorem 1.1,

$$
\frac{1}{\Gamma(2-\gamma)}=\lim _{\substack{r \rightarrow \infty(r \rightarrow 0, \text { resp. }) \\ t \phi\left(r^{-1}\right) \rightarrow 0}} \frac{\mathbb{P}\left(T_{t} \geq r\right)}{t \phi\left(r^{-1}\right)} \cdot \frac{\phi\left(r^{-1}\right)}{H\left(r^{-1}\right)}=\frac{1}{1-\gamma} \lim _{\substack{r \rightarrow \infty(r \rightarrow 0, \text { resp. }) \\ t \phi\left(r^{-1}\right) \rightarrow 0}} \frac{\mathbb{P}\left(T_{t} \geq r\right)}{t \phi\left(r^{-1}\right)}
$$

yielding the desired estimate.

The following proposition explains the decay of the tail of the Lévy measure of the subordinator $T$.

Proposition 2.3. Assume that $H(\lambda)=\phi(\lambda)-\lambda \phi^{\prime}(\lambda)$ varies regularly at 0 (at $\infty$, respectively) with index $\gamma \in[0,1]$. Then

$$
\mu(r, \infty) \sim \frac{1}{\Gamma(2-\gamma)} H\left(r^{-1}\right), \quad r \rightarrow \infty(r \rightarrow 0, \text { resp. })
$$

Proof. It is easy to show that

$$
b+\int_{0}^{\infty} e^{-\lambda r} \mu(r, \infty) d r=\frac{\phi(\lambda)}{\lambda} .
$$

Taking the derivative we obtain

$$
\int_{0}^{\infty} e^{-\lambda r} r \mu(r, \infty) d r=\frac{H(\lambda)}{\lambda^{2}}
$$

Since

$$
\begin{aligned}
\lambda^{-2} & =\lim _{\substack{x \rightarrow \infty \\
x \rightarrow 0 \text { resp. })}} \int_{0}^{\infty} e^{-\lambda x^{-1} r} \frac{r \mu(r, \infty)}{x^{2} H\left(\lambda x^{-1}\right)} d r \\
& =\lim _{\substack{x \rightarrow \infty \\
(x \rightarrow 0 \text { resp. })}} \int_{0}^{\infty} e^{-\lambda r} \frac{r \mu(r x, \infty)}{H\left(x^{-1}\right)} d r \frac{H\left(x^{-1}\right)}{H\left(\lambda x^{-1}\right)},
\end{aligned}
$$

we can use regular varation of $H$ to get

$$
\lim _{\substack{x \rightarrow \infty \\(x \rightarrow 0 \text { resp. })}} \int_{0}^{\infty} e^{-\lambda r} \frac{r \mu(r x, \infty)}{H\left(x^{-1}\right)} d r=\lambda^{\gamma-2} .
$$

Note that the last display is very similar to (2.7). We now repeat the argument after (2.7) in the proof of Theorem 1.1 to obtain the claim.

\section{Asymptotic of the tail probability of isotropic LÉvy processes}

Throughout this section, we assume that $X=\left(X_{t}\right)_{t \geq 0}$ is an isotropic Lévy process in $\mathbb{R}^{d}(d \geq 1)$ with the characteristic exponent $\xi \rightarrow \psi(|\xi|)$. The following is known and, in fact true for any negative definite function (see [Grz14, Lemma 1]). 
Lemma 3.1. For every $t>0$ and $\lambda \geq 1$,

$$
\psi^{*}(\lambda t) \leq 2\left(1+\lambda^{2}\right) \psi^{*}(t)
$$

where $\psi^{*}(r):=\sup _{|z| \leq r} \psi(|z|)$.

We start with

$$
e^{-|x|^{2}}=(4 \pi)^{-d / 2} \int_{\mathbb{R}^{d}} e^{i \xi \cdot x} e^{-\frac{|\xi|^{2}}{4}} d \xi
$$

to obtain the Laplace transform of the measure $\mathbb{P}\left(\left|X_{t}\right| \geq \sqrt{r}\right) d r$ (see also [CGT15]);

$$
\begin{aligned}
\int_{0}^{\infty} e^{-\lambda r} \mathbb{P}\left(\left|X_{t}\right| \geq \sqrt{r}\right) d r & =\mathbb{E} \int_{0}^{\left|X_{t}\right|^{2}} e^{-\lambda r} d r=(4 \pi)^{-d / 2} \int_{\mathbb{R}^{d}} \frac{1-\mathbb{E}\left[e^{i \sqrt{\lambda \xi} \cdot X_{t}}\right]}{\lambda} e^{-\frac{|\xi|^{2}}{4}} d \xi \\
& =(4 \pi)^{-d / 2} \int_{\mathbb{R}^{d}} \frac{1-e^{-t \psi(|\xi| \sqrt{\lambda})}}{\lambda} e^{-\frac{|\xi|^{2}}{4}} d \xi \\
& =\frac{2^{1-d}}{\Gamma(d / 2)} \int_{0}^{\infty} \frac{1-e^{-t \psi(r \sqrt{\lambda})}}{\lambda} e^{-\frac{r^{2}}{4}} r^{d-1} d r \\
& =\frac{2^{1-d}}{\Gamma(d / 2)} \int_{0}^{\infty} \lambda^{-d / 2-1} e^{-\frac{r^{2}}{4 \lambda}}\left(1-e^{-t \psi(r)}\right) r^{d-1} d r
\end{aligned}
$$

for all $\lambda>0$.

One of the ideas of proving the asymptotic behaviors of the tail probability and the transition density is using the following function $\phi$, which is nicer than $\psi$. Define the auxiliary function $\phi:(0, \infty) \rightarrow(0, \infty)$ by

$$
\phi(\lambda)=(4 \pi)^{-d / 2} \int_{\mathbb{R}^{d}} e^{-\frac{|\xi|^{2}}{4}} \psi(|\xi| \sqrt{\lambda}) d \xi, \quad \lambda>0 .
$$

The following forms of $\phi$ are useful in evaluation of its derivative (since then we do not have to take derivative of $\psi$ );

$$
\phi(\lambda)=\int_{\mathbb{R}^{d}}(4 \pi \lambda)^{-d / 2} e^{-\frac{|\xi|^{2}}{4 \lambda}} \psi(|\xi|) d \xi=\frac{2^{1-d}}{\Gamma(d / 2)} \int_{0}^{\infty} \lambda^{-d / 2} e^{-\frac{r^{2}}{4 \lambda}} \psi(r) r^{d-1} d r .
$$

Remark 3.2. The function $\phi$ is, in fact, a Bernstein function and the Laplace exponent of a subordinator. Moreover, if $a=0$ in (1.5) then $\phi$ is the Laplace exponent of a subordinator without drift. 
Indeed, by using (1.5), (3.1) and polar coordinates, we obtain

$$
\begin{aligned}
\phi(\lambda) & =a \int_{\mathbb{R}^{d}}(4 \pi \lambda)^{-d / 2} e^{-\frac{|\xi|^{2}}{4 \lambda}}|\xi|^{2} d \xi+\int_{\mathbb{R}^{d}} \int_{\mathbb{R}^{d}}(4 \pi \lambda)^{-d / 2} e^{-\frac{|\xi|^{2}}{4 \lambda}}\left(1-e^{i \xi \cdot x}\right) d \xi \nu(d x) \\
& =a \sum_{i=1}^{d} \int_{\mathbb{R}^{d}}(4 \pi \lambda)^{-d / 2} e^{-\frac{|\xi|^{2}}{4 \lambda}} \xi_{i}^{2} d \xi+\int_{\mathbb{R}^{d}}\left(1-e^{-\lambda|x|^{2}}\right) \nu(d x) \\
& =a \sum_{i=1}^{d} \int_{\mathbb{R}^{-}}(4 \pi \lambda)^{-1 / 2} e^{-\frac{\xi_{i}^{2}}{4 \lambda}} \xi_{i}^{2} d \xi+\int_{0}^{\infty}\left(1-e^{-\lambda r^{2}}\right) \int_{\partial B(0, r)} \nu(d y) d r \\
& =2 a \lambda d+2^{-1} \int_{0}^{\infty}\left(1-e^{-\lambda r}\right) r^{-1 / 2} \int_{\partial B(0, \sqrt{r})} \nu(d y) d r .
\end{aligned}
$$

Denoting $\mu(d r)=\frac{1}{2 \sqrt{r}} \int_{\partial B(0, \sqrt{r})} \nu(d y) d r$, we obtain that $\mu$ is the Lévy measure of a subordinator, since

$$
\begin{aligned}
\int_{0}^{\infty}(1 \wedge r) \mu(d r) & =\int_{0}^{\infty}(1 \wedge r) \frac{1}{2 \sqrt{r}} \int_{\partial B(0, \sqrt{r})} \nu(d y) d r \\
& =\int_{0}^{\infty}\left(1 \wedge r^{2}\right) \int_{\partial B(0, r)} \nu(d y) d r=\int_{\mathbb{R}^{d}}\left(1 \wedge|x|^{2}\right) \nu(d x)<\infty
\end{aligned}
$$

As in the subordinate Brownian motion case, set

$$
H(\lambda)=\phi(\lambda)-\lambda \phi^{\prime}(\lambda), \quad \lambda>0 .
$$

By Remark 3.2, $H$ is non-negative and non-decreasing.

Using (3.5), we obtain $H$ in terms of $\psi$ as

$$
\begin{aligned}
H(\lambda) & =-\lambda^{2}\left(\frac{\phi(\lambda)}{\lambda}\right)^{\prime}= \\
& =\frac{2^{1-d}}{\Gamma\left(\frac{d}{2}\right)} \int_{0}^{\infty} \lambda^{-d / 2} e^{-\frac{r^{2}}{4 \lambda}}\left(-\frac{r^{2}}{4 \lambda}+\frac{d+2}{2}\right) \psi(r) r^{d-1} d r .
\end{aligned}
$$

We now discuss compatibilities of functions $\phi(\lambda), H(\lambda)$ and $\psi(\sqrt{\lambda})$.

Proposition 3.3. Assume that $\psi$ varies regularly at 0 (at $\infty$, respectively) with index $\alpha \in[0,2]$. Then

$$
\phi(\lambda) \sim 2^{\alpha} \frac{\Gamma\left(\frac{d+\alpha}{2}\right)}{\Gamma\left(\frac{d}{2}\right)} \psi(\sqrt{\lambda}), \quad \lambda \rightarrow 0(\lambda \rightarrow \infty, \text { resp. })
$$


(2)

$$
\lim _{\lambda \rightarrow 0} \frac{H(\lambda)}{\psi(\sqrt{\lambda})}=2^{\alpha-1}(2-\alpha) \frac{\Gamma\left(\frac{d+\alpha}{2}\right)}{\Gamma\left(\frac{d}{2}\right)}
$$

where $\phi$ is defined in (3.5). Consequently, $\phi$ varies regularly at 0 (at $\infty$, respectively) with index $\alpha / 2$ and, if $\alpha<2$, then $H$ varies regularly at 0 (at $\infty$, respectively) with index $\alpha / 2$.

Proof. (1) By (3.5) we have

$$
\begin{aligned}
\frac{\phi(\lambda)}{\psi(\sqrt{\lambda})} & =\frac{1}{2^{d-1} \Gamma\left(\frac{d}{2}\right)}\left(\int_{0}^{1 / \sqrt{\lambda}} e^{-\frac{r^{2}}{4}} r^{d-1} \frac{\psi(r \sqrt{\lambda})}{\psi(\sqrt{\lambda})} d r+\int_{1 / \sqrt{\lambda}}^{\infty} e^{-\frac{r^{2}}{4}} r^{d-1} \frac{\psi(r \sqrt{\lambda})}{\psi(\sqrt{\lambda})} d r\right) \\
& =I(\lambda)+\frac{1}{2^{d-1} \Gamma\left(\frac{d}{2}\right)} I I(\lambda) .
\end{aligned}
$$

We first assume that $\psi$ varies regularly at 0 with index $\alpha \in[0,2]$. By Potter's theorem (see [BGT87, Theorem 1.5.6]) there exists a constant $c_{1}>0$ such that

$$
\frac{\psi(r \sqrt{\lambda})}{\psi(\sqrt{\lambda})} \leq c_{1}\left(r^{3} \vee r^{-1 / 2}\right), \quad \text { for } 1>r \sqrt{\lambda}>0,1>\lambda>0 .
$$

So, by the dominated convergence theorem we have

$$
\begin{aligned}
\lim _{\lambda \downarrow 0} I(\lambda) & =\frac{1}{2^{d-1} \Gamma\left(\frac{d}{2}\right)} \int_{0}^{\infty} e^{-\frac{r^{2}}{4}} r^{\alpha+d-1} d r \\
& =\frac{1}{2^{d-1} \Gamma\left(\frac{d}{2}\right)} \int_{0}^{\infty} e^{-r} 2^{\alpha+d-1} r^{\frac{d+\alpha}{2}-1} d r=2^{\alpha} \frac{\Gamma\left(\frac{d+\alpha}{2}\right)}{\Gamma\left(\frac{d}{2}\right)} .
\end{aligned}
$$

By Lemma 3.1 we have $\int_{1}^{\infty} e^{-\frac{r^{2}}{8}} \psi(r) r^{-5} d r=: c_{2}<\infty$. Thus $\lambda \in(0,1]$

$$
\begin{aligned}
& \lambda^{-d / 2} \int_{1}^{\infty} e^{-\frac{r^{2}}{4 \lambda}} r^{d-1} \psi(r) d r=\lambda^{2} \int_{1}^{\infty}\left(\frac{r}{\sqrt{\lambda}}\right)^{d+4} e^{-\frac{r^{2}}{4 \lambda}} \psi(r) r^{-5} d r \\
& \leq \lambda^{2} \int_{1}^{\infty}\left(\frac{r}{\sqrt{\lambda}}\right)^{d+4} e^{-\frac{r^{2}}{4 \lambda}} \psi(r) r^{-5} d r \\
& \leq \lambda^{2}\left(\sup _{y \geq 1} y^{d+4} e^{-y / 8}\right) \int_{1}^{\infty} e^{-\frac{r^{2}}{8}} \psi(r) r^{-5} d r=: c_{3} \lambda^{2} .
\end{aligned}
$$

Since $\lambda \mapsto \psi(\sqrt{\lambda})$ varies regularly with index $\alpha / 2 \in[0,1]$, after change of variable, we have that

$$
\limsup _{\lambda \downarrow 0} I I(\lambda)=\limsup _{\lambda \downarrow 0} \frac{\lambda^{-d / 2}}{\psi(\sqrt{\lambda})} \int_{1}^{\infty} e^{-\frac{r^{2}}{4 \lambda}} r^{d-1} \psi(r) d r \leq c_{3} \limsup _{\lambda \downarrow 0} \frac{\lambda^{2}}{\psi(\sqrt{\lambda})}=0 .
$$


Therefore,

$$
\lim _{\lambda \downarrow 0} \frac{\phi(\lambda)}{\psi(\sqrt{\lambda})}=\frac{1}{2^{d} \Gamma\left(\frac{d}{2}\right)} \lim _{\lambda \downarrow 0} I(\lambda)=2^{\alpha} \frac{\Gamma\left(\frac{d+\alpha}{2}\right)}{\Gamma\left(\frac{d}{2}\right)} .
$$

We now assume that $\psi$ varies regularly at $\infty$ with index $\alpha \in[0,2]$. In this case, through analogous argument

$$
\lim _{\lambda \uparrow \infty} I I(\lambda)=2^{\alpha} \frac{\Gamma\left(\frac{d+\alpha}{2}\right)}{\Gamma\left(\frac{d}{2}\right)}
$$

Moreover, by change of variable

$$
I(\lambda)=\frac{1}{\lambda^{d / 2} \psi(\sqrt{\lambda})} \int_{0}^{1} e^{-\frac{r^{2}}{4 \lambda}} r^{d-1} \psi(r) d r \leq \frac{1}{\lambda^{d / 2} \psi(\sqrt{\lambda})} \int_{0}^{1} r^{d-1} \psi(r) d r
$$

which goes to zero as $\lambda \uparrow \infty$.

(2) As we observed in (1), the proof will be analogous and simpler when $\psi$ varies regularly at $\infty$ with index $\alpha \in[0,2]$. Thus we only provide the proof for the case that $\psi$ varies regularly at 0 with index $\alpha \in[0,2]$.

Using (3.12) and change of variable, we rewrite as

$$
\begin{aligned}
& \frac{H(\lambda)}{\psi(\sqrt{\lambda})}=\frac{2^{1-d}}{\Gamma\left(\frac{d}{2}\right)} \int_{0}^{\infty} \lambda^{-d / 2} e^{-\frac{r^{2}}{4 \lambda}}\left(\frac{d+2}{2}-\frac{r^{2}}{4 \lambda}\right) r^{d-1} \frac{\psi(r)}{\psi(\sqrt{\lambda})} d r \\
&=\frac{2^{1-d}}{\Gamma\left(\frac{d}{2}\right)} \int_{0}^{1} \lambda^{-d / 2} e^{-\frac{r^{2}}{4 \lambda}}\left(\frac{d+2}{2}-\frac{r^{2}}{4 \lambda}\right) r^{d-1} \frac{\psi(r)}{\psi(\sqrt{\lambda})} d r \\
& \quad+\frac{2^{1-d}}{\Gamma\left(\frac{d}{2}\right)} \int_{1}^{\infty} \lambda^{-d / 2} e^{-\frac{r^{2}}{4 \lambda}}\left(\frac{d+2}{2}-\frac{r^{2}}{4 \lambda}\right) r^{d-1} \frac{\psi(r)}{\psi(\sqrt{\lambda})} d r \\
&=\frac{1}{\Gamma\left(\frac{d}{2}\right)} \int_{0}^{(4 \lambda)^{-1}} e^{-r} r^{\frac{d}{2}-1}\left(\frac{d+2}{2}-r\right) \frac{\psi(2 \sqrt{r} \sqrt{\lambda})}{\psi(\sqrt{\lambda})} d r \\
& \quad+\frac{2^{1-d}}{\Gamma\left(\frac{d}{2}\right)} \frac{1}{\psi(\sqrt{\lambda})} \int_{1}^{\infty} \lambda^{-d / 2} e^{-\frac{r^{2}}{4 \lambda}}\left(\frac{d+2}{2}-\frac{r^{2}}{4 \lambda}\right) r^{d-1} \psi(r) d r \\
&= I(\lambda)+\frac{2^{1-d}}{\Gamma\left(\frac{d}{2}\right)} I I(\lambda) .
\end{aligned}
$$


Similar to (1), by Potter's theorem as (3.7) we can use the dominated convergence theorem and get

$$
\begin{aligned}
\lim _{\lambda \downarrow 0} I(\lambda) & =\frac{1}{\Gamma\left(\frac{d}{2}\right)} \int_{0}^{\infty} e^{-r} r^{\frac{d}{2}-1}\left(\frac{d+2}{2}-r\right) 2^{\alpha} r^{\frac{\alpha}{2}} d r \\
& =\frac{2^{\alpha}}{\Gamma\left(\frac{d}{2}\right)}\left(\frac{d+2}{2} \Gamma\left(\frac{d+\alpha}{2}\right)-\Gamma\left(\frac{d+\alpha}{2}+1\right)\right) \\
& =\frac{2^{\alpha}}{\Gamma\left(\frac{d}{2}\right)}\left(\frac{d+2}{2} \Gamma\left(\frac{d+\alpha}{2}\right)-\frac{d+\alpha}{2} \Gamma\left(\frac{d+\alpha}{2}\right)\right) \\
& =2^{\alpha-1}(2-\alpha) \frac{\Gamma\left(\frac{d+\alpha}{2}\right)}{\Gamma\left(\frac{d}{2}\right)} .
\end{aligned}
$$

Similar as (3.8) and (3.9), for $\lambda \in(0,1]$ we also bound $I I(\lambda)$ as

$$
\begin{aligned}
& |I I(\lambda)| \leq \frac{1}{\psi(\sqrt{\lambda})} \int_{1}^{\infty} \lambda^{-d / 2} e^{-\frac{r^{2}}{4 \lambda}}\left(\frac{d+2}{2}+\frac{r^{2}}{4 \lambda}\right) r^{d-1} \psi(r) d r \\
& \leq \frac{2 d+5}{4} \frac{1}{\psi(\sqrt{\lambda})} \int_{1}^{\infty} \lambda^{-d / 2-1} e^{-\frac{r^{2}}{4 \lambda}} r^{d+1} \psi(r) d r \\
& =\frac{2 d+5}{4} \frac{\lambda^{2}}{\psi(\sqrt{\lambda})} \int_{1}^{\infty}\left(\frac{r}{\sqrt{\lambda}}\right)^{d+6} e^{-\frac{r^{2}}{4 \lambda}} r^{-5} \psi(r) d r \\
& \leq \frac{2 d+5}{4} \frac{\lambda^{2}}{\psi(\sqrt{\lambda})}\left(\sup _{y \geq 1} y^{d+6} e^{-y / 8}\right) \int_{1}^{\infty} e^{-\frac{r^{2}}{8}} r^{-5} \psi(r) d r
\end{aligned}
$$

which goes to zero as $\lambda \rightarrow 0$.

Therefore,

$$
\lim _{\lambda \downarrow 0} \frac{H(\lambda)}{\psi(\sqrt{\lambda})}=2^{\alpha-1}(2-\alpha) \frac{\Gamma\left(\frac{d+\alpha}{2}\right)}{\Gamma\left(\frac{d}{2}\right)}
$$

It follows from the previous result that, in the case $\alpha=2$, functions $H(\lambda)$ and $\psi(\sqrt{\lambda})$ are not comparable. We clarify this situation in the following result.

Proposition 3.4. Let $g(\lambda)=\psi(\lambda)-\frac{1}{2} \lambda \psi^{\prime}(\lambda)$. If $g$ varies reularly at 0 (at $\infty$, respectively) with index $\alpha \geq 0$, then

$$
H(\lambda)=\phi(\lambda)-\lambda \phi^{\prime}(\lambda) \sim \frac{2^{\alpha} \Gamma\left(\frac{d+\alpha}{2}\right)}{\Gamma\left(\frac{d}{2}\right)} g(\sqrt{\lambda}), \quad \lambda \rightarrow 0(\lambda \rightarrow \infty, \text { resp. }) .
$$


Proof. Since the proof for the case that $g$ varies regularly at $\infty$ will be analogous and simpler, we only provide the proof for the case that $g$ varies regularly at 0 with index $\alpha \geq 0$.

Note that, by the change of variable we have

$$
\begin{aligned}
\phi(\lambda) & =\frac{2^{1-d}}{\Gamma\left(\frac{d}{2}\right)}\left(\int_{0}^{1 / \sqrt{\lambda}} e^{-r^{2} / 4} r^{d-1} \psi(r \sqrt{\lambda}) d r+\int_{1 / \sqrt{\lambda}}^{\infty} e^{-r^{2} / 4} r^{d-1} \psi(r \sqrt{\lambda}) d r\right) \\
& =\frac{2^{1-d}}{\Gamma\left(\frac{d}{2}\right)}\left(\int_{0}^{1 / \sqrt{\lambda}} e^{-r^{2} / 4} r^{d-1} \psi(r \sqrt{\lambda}) d r+\lambda^{-d / 2} \int_{1}^{\infty} r^{d-1} e^{-\frac{r^{2}}{4 \lambda}} \psi(r) d r\right) .
\end{aligned}
$$

By Potter's theorem (see [BGT87, Theorem 1.5.6]) there exists a constant $c_{0}>0$ such that

$$
g(r \sqrt{\lambda}) \leq c_{0} g(1)(r \sqrt{\lambda})^{-1 / 2}, \quad \text { for } 1>r \sqrt{\lambda}>0,
$$

Thus we can differentiate under the integral sign and get

$$
\begin{aligned}
& \left(\int_{0}^{1 / \sqrt{\lambda}} e^{-r^{2} / 4} r^{d-1} \lambda^{-1} \psi(r \sqrt{\lambda}) d r\right)^{\prime} \\
= & e^{-1 /(4 \lambda)} \lambda^{-(d-1) / 2} \lambda^{-1} \psi(1)\left(-\frac{1}{2} \lambda^{-3 / 2}\right)+\int_{0}^{1 / \sqrt{\lambda}} e^{-r^{2} / 4} r^{d-1} \frac{\partial}{\partial \lambda}\left(\frac{\psi(r \sqrt{\lambda})}{\lambda}\right) d r \\
= & -\frac{1}{2} e^{-1 /(4 \lambda)} \lambda^{-2-d / 2} \psi(1)-\int_{0}^{1 / \sqrt{\lambda}} e^{-r^{2} / 4} r^{d-1} \frac{g(r \sqrt{\lambda})}{\lambda^{2}} d r .
\end{aligned}
$$

From (3.10) and (3.11) we obtain that

$$
\begin{aligned}
H(\lambda)=-\lambda^{2}\left(\frac{\phi(\lambda)}{\lambda}\right)^{\prime} \\
=-\frac{2^{1-d}}{\Gamma\left(\frac{d}{2}\right)} \lambda^{2}\left(\int_{0}^{1 / \sqrt{\lambda}} e^{-r^{2} / 4} r^{d-1} \lambda^{-1} \psi(r \sqrt{\lambda}) d r+\lambda^{-1-d / 2} \int_{1}^{\infty} r^{d-1} e^{-\frac{r^{2}}{4 \lambda}} \psi(r) d r\right)^{\prime} \\
=-\frac{2^{1-d}}{\Gamma\left(\frac{d}{2}\right)} \lambda^{2}\left(-\frac{1}{2} e^{-1 /(4 \lambda)} \lambda^{-2-d / 2} \psi(1)-\int_{0}^{1 / \sqrt{\lambda}} e^{-r^{2} / 4} r^{d-1} \frac{g(r \sqrt{\lambda})}{\lambda^{2}} d r\right. \\
\left.\quad+\int_{1}^{\infty} \lambda^{-2-d / 2} e^{-\frac{r^{2}}{4 \lambda}}\left(\frac{r^{2}}{4 \lambda}-\frac{d+2}{2}\right) \psi(r) r^{d-1} d r\right) \\
=\frac{2^{1-d}}{\Gamma\left(\frac{d}{2}\right)}\left(\int_{0}^{1 / \sqrt{\lambda}} e^{-r^{2} / 4} r^{d-1} g(r \sqrt{\lambda}) d r+\frac{1}{2} e^{-1 /(4 \lambda)} \lambda^{-d / 2} \psi(1)\right.
\end{aligned}
$$




$$
\left.+\int_{1}^{\infty} \lambda^{-d / 2} e^{-\frac{r^{2}}{4 \lambda}}\left(-\frac{r^{2}}{4 \lambda}+\frac{d+2}{2}\right) \psi(r) r^{d-1} d r\right)
$$

To evaluate the limit we use the above form so that

$$
\begin{aligned}
& \frac{H(\lambda)}{g(\sqrt{\lambda})}=\frac{2^{1-d}}{\Gamma\left(\frac{d}{2}\right)} \int_{0}^{1 / \sqrt{\lambda}} e^{-r^{2} / 4} r^{d-1} \frac{g(r \sqrt{\lambda})}{g(\sqrt{\lambda})} d r \\
& +\frac{2^{1-d}}{\Gamma\left(\frac{d}{2}\right) g(\sqrt{\lambda})}\left(\frac{1}{2} e^{-1 /(4 \lambda)} \lambda^{-d / 2} \psi(1)+\int_{1}^{\infty} \lambda^{-d / 2} e^{-\frac{r^{2}}{4 \lambda}}\left(-\frac{r^{2}}{4 \lambda}+\frac{d+2}{2}\right) \psi(r) r^{d-1} d r\right) \\
& =I_{1}(\lambda)+\frac{2^{1-d}}{\Gamma\left(\frac{d}{2}\right) g(\sqrt{\lambda})} I_{2}(\lambda) .
\end{aligned}
$$

By Potter's theorem (see [BGT87, Theorem 1.5.6]) there exists a constant $c_{1}>0$ such that

$$
\frac{g(r \sqrt{\lambda})}{g(\sqrt{\lambda})} \leq c_{1}\left(r^{\alpha+1} \vee r^{-1 / 2}\right), \quad \text { for } 1>r \sqrt{\lambda}>0,1>\lambda>0
$$

and so we may use dominated convergence theorem in the first integral to obtain

$$
\lim _{\lambda \downarrow 0} I_{1}(\lambda)=\frac{2^{1-d}}{\Gamma\left(\frac{d}{2}\right)} \int_{0}^{\infty} e^{-r^{2} / 4} r^{d+\alpha-1} d r=\frac{2^{\alpha}}{\Gamma\left(\frac{d}{2}\right)} \int_{0}^{\infty} e^{-r} r^{\frac{d+\alpha}{2}-1} d r=\frac{2^{\alpha} \Gamma\left(\frac{d+\alpha}{2}\right)}{\Gamma\left(\frac{d}{2}\right)} .
$$

On the other hand, we can rewrite $I_{2}$ as

$$
\begin{aligned}
I_{2}(\lambda)= & (1+d / 2) \lambda^{-d / 2} \int_{1}^{\infty} r^{d-1} e^{-\frac{r^{2}}{4 \lambda}} \psi(r) d r \\
& +\frac{1}{2} \lambda^{-d / 2} e^{-\frac{1}{4 \lambda}} \psi(1)-\frac{1}{4} \lambda^{-1-d / 2} \int_{1}^{\infty} r^{d+1} e^{-\frac{r^{2}}{4 \lambda}} \psi(r) d r \\
= & \lambda^{(1+\alpha) / 2} 2\left((1+d / 2) \int_{1}^{\infty}\left(\frac{r}{\sqrt{\lambda}}\right)^{d+1+\alpha} e^{-\frac{r^{2}}{4 \lambda}} \psi(r) r^{-2-\alpha} d r\right. \\
& \left.+\frac{1}{2} \lambda^{-(d+1+\alpha) / 2} e^{-\frac{1}{4 \lambda}} \psi(1)-\frac{1}{4} \int_{1}^{\infty}\left(\frac{r}{\sqrt{\lambda}}\right)^{d+3+\alpha} e^{-\frac{r^{2}}{4 \lambda}} \psi(r) r^{-2-\alpha} d r\right)
\end{aligned}
$$

Thus for $\lambda \in(0,1]$

$$
\begin{aligned}
& \left|I_{2}(\lambda)\right| \leq c_{2} \lambda^{(1+\alpha) / 2}\left(\lambda^{-(d+1+\alpha) / 2} e^{-\frac{1}{4 \lambda}}+\int_{1}^{\infty}\left(\frac{r}{\sqrt{\lambda}}\right)^{d+3+\alpha} e^{-\frac{r^{2}}{4 \lambda}} \psi(r) r^{-2-\alpha} d r\right) \\
& \leq c_{2} \lambda^{(1+\alpha) / 2}\left(\left(\sup _{y \geq 1} y^{(d+1+\alpha) / 2} e^{-y / 4}\right)+\left(\sup _{y \geq 1} y^{d+3+\alpha} e^{-y / 8}\right) \int_{1}^{\infty} e^{-\frac{r^{2}}{8}} \psi(r) r^{-2-\alpha} d r\right) \\
& \leq c_{3} \lambda^{(1+\alpha) / 2},
\end{aligned}
$$


where in the last inequality we have used $\psi(r) \leq 2\left(1+r^{2}\right) \sup _{s \leq 1} \psi(s)$ for all $r>0$ by Lemma 3.1. Since $\lambda \mapsto g(\sqrt{\lambda})$ varies regularly with index $\alpha / 2$ we now conclude that

$$
\limsup _{\lambda \downarrow 0} \frac{\left|I_{2}(\lambda)\right|}{g(\sqrt{\lambda})} \leq c_{3} \limsup _{\lambda \downarrow 0} \frac{\lambda^{(1+\alpha) / 2}}{g(\sqrt{\lambda})}=0 .
$$

Lemma 3.5. Assume that $H(\lambda)=\phi(\lambda)-\lambda \phi^{\prime}(\lambda)$ varies regularly at 0 (at $\infty$, respectively) with index $\gamma \in[0,2)$ and $\psi$ varies regularly at 0 (at $\infty$, respectively) with index $\gamma_{1} \in[0,2]$. Then for all $R>0$ we have

$$
\begin{gathered}
\int_{0}^{R} r \mathbb{P}\left(\left|X_{t}\right| \geq \sqrt{r} \sqrt{s}\right) d r \sim \frac{R^{2-\gamma}}{\Gamma(3-\gamma)} t H\left(s^{-1}\right), \quad s \rightarrow \infty(s \rightarrow 0, \text { resp. }) \\
\text { and } t \frac{\psi\left(\sqrt{s}^{-1}\right)^{2}}{H\left(s^{-1}\right)} \rightarrow 0 .
\end{gathered}
$$

Proof. We provide the proof for the case that $H$ varies regularly at 0 with index $\gamma \in[0,2)$. The proof of the other case is similar.

Taking derivative in (3.3) we obtain

$$
\begin{array}{rl}
\int_{0}^{\infty} e^{-\lambda r} r & \mathbb{P}\left(\left|X_{t}\right| \geq \sqrt{r}\right) d r \\
& =\frac{2^{1-d}}{\Gamma(d / 2)} \int_{0}^{\infty} \lambda^{-d / 2-2} e^{-\frac{r^{2}}{4 \lambda}}\left(\frac{d+2}{2}-\frac{r^{2}}{4 \lambda}\right)\left(1-e^{-t \psi(r)}\right) r^{d-1} d r .
\end{array}
$$

Since we have from (3.12)

$$
\frac{H(\lambda)}{\lambda^{2}}=\frac{2^{1-d}}{\Gamma(d / 2)} \int_{0}^{\infty} \lambda^{-d / 2-2} e^{-\frac{r^{2}}{4 \lambda}}\left(\frac{d+2}{2}-\frac{r^{2}}{4 \lambda}\right) \psi(r) r^{d-1} d r,
$$

comparing the above two displays and changing variable we see that

$$
\begin{aligned}
\int_{0}^{\infty} e^{-\lambda r} r \mathbb{P}\left(\left|X_{t}\right| \geq \sqrt{r}\right) d r=\frac{t H(\lambda)}{\lambda^{2}} \\
\quad-\frac{2^{1-d}}{\Gamma(d / 2)} \int_{0}^{\infty} e^{-\frac{r^{2}}{4}}\left(\frac{r^{2}}{4}-\frac{d+2}{2}\right) \frac{1-e^{-t \psi(r \sqrt{\lambda})}-t \psi(r \sqrt{\lambda})}{\lambda^{2}} r^{d-1} d r .
\end{aligned}
$$


We replace $\lambda$ in (3.13) by $\lambda s^{-1}$ and divide both sides by $t H\left(s^{-1}\right)$ and get

$$
\begin{aligned}
& \int_{0}^{\infty} r e^{-\lambda s^{-1} r} \frac{\mathbb{P}\left(\left|X_{t}\right| \geq \sqrt{r}\right)}{t H\left(s^{-1}\right)} d r=s^{2} \frac{H\left(\lambda s^{-1}\right)}{\lambda^{2} H\left(s^{-1}\right)} \\
& \quad-s^{2} \frac{2^{1-d}}{\Gamma(d / 2)} \int_{0}^{\infty} e^{-\frac{r^{2}}{4}}\left(\frac{r^{2}}{4}-\frac{d+2}{2}\right) \frac{1-e^{-t \psi(r \sqrt{\lambda} \sqrt{s}-1)}-t \psi\left(r \sqrt{\lambda} \sqrt{s}{ }^{-1}\right)}{\lambda^{2} t H\left(s^{-1}\right)} r^{d-1} d r .
\end{aligned}
$$

Now changing variable in the first integral yields

$$
\begin{aligned}
& \int_{0}^{\infty} r e^{-\lambda r} \frac{\mathbb{P}\left(\left|X_{t}\right| \geq \sqrt{r} \sqrt{s}\right)}{t H\left(s^{-1}\right)} d r=\frac{H\left(\lambda s^{-1}\right)}{\lambda^{2} H\left(s^{-1}\right)} \\
& -\frac{2^{1-d}}{\Gamma(d / 2)} \int_{0}^{\infty} e^{-\frac{r^{2}}{4}}\left(\frac{r^{2}}{4}-\frac{d+2}{2}\right) \frac{1-e^{-t \psi\left(r \sqrt{\lambda} \sqrt{s}^{-1}\right)}-t \psi\left(r \sqrt{\lambda} \sqrt{s}{ }^{-1}\right)}{\lambda^{2} t H\left(s^{-1}\right)} r^{d-1} d r .
\end{aligned}
$$

Note that, since

$$
\begin{aligned}
\frac{\left|1-e^{-t \psi\left(r \sqrt{\lambda} \sqrt{s}^{-1}\right)}-t \psi\left(r \sqrt{\lambda} \sqrt{s}^{-1}\right)\right|}{t H\left(s^{-1}\right)} & \leq \frac{1}{2} \frac{t \psi\left(r \sqrt{\lambda} \sqrt{s}^{-1}\right)^{2}}{H\left(s^{-1}\right)} \\
& =\frac{1}{2}\left(\frac{\psi\left(r \sqrt{\lambda} \sqrt{s}^{-1}\right)}{\psi\left(\sqrt{s}^{-1}\right)}\right)^{2} \frac{t \psi\left(\sqrt{s}^{-1}\right)^{2}}{H\left(s^{-1}\right)}
\end{aligned}
$$

we have that for $s \geq \lambda$

$$
\begin{aligned}
& \left|\int_{0}^{\infty} e^{-\frac{r^{2}}{4}}\left(\frac{r^{2}}{4}-\frac{d+2}{2}\right) \frac{1-e^{-t \psi\left(r \sqrt{\lambda} \sqrt{s}^{-1}\right)}-t \psi\left(r \sqrt{\lambda} \sqrt{s}^{-1}\right)}{\lambda^{2} t H\left(s^{-1}\right)} r^{d-1} d r\right| \\
\leq & \frac{1}{2} \frac{t \psi\left(\sqrt{s}^{-1}\right)^{2}}{H\left(s^{-1}\right)} \int_{0}^{\infty} e^{-\frac{r^{2}}{4}}\left|\frac{r^{2}}{4}-\frac{d+2}{2}\right|\left(\frac{\psi(r \sqrt{\lambda} \sqrt{s}-1)}{\psi\left(\sqrt{s}^{-1}\right)}\right)^{2} r^{d-1} d r \\
\leq & \frac{1}{2} \frac{t \psi\left(\sqrt{s}^{-1}\right)^{2}}{H\left(s^{-1}\right)}\left(\int_{0}^{\sqrt{s} \sqrt{\lambda}^{-1}} e^{-\frac{r^{2}}{4}}\left|\frac{r^{2}}{4}-\frac{d+2}{2}\right|\left(\frac{\psi\left(r \sqrt{\lambda} \sqrt{s}^{-1}\right)}{\left.\psi(\sqrt{s})^{-1}\right)}\right)^{2} r^{d-1} d r\right. \\
& \left.+\frac{2 d+5}{4} \int_{\sqrt{s} \sqrt{\lambda}}^{\infty} e^{-\frac{r^{2}}{4}}\left(\frac{\psi\left(r \sqrt{\lambda} \sqrt{s}^{-1}\right)}{\psi(\sqrt{s}-1)}\right)^{2} r^{d+1} d r\right) \\
= & : \frac{1}{2} \frac{t \psi(\sqrt{s})^{2}}{H\left(s^{-1}\right)}\left(I(s)+\frac{2 d+5}{4} I I(s)\right) .
\end{aligned}
$$

Since $\psi$ varies regularly at 0 with index $\gamma_{1}$, by Potter's theorem (see [BGT87, Theorem 1.5.6]) it follows that, for any $\varepsilon>0$ there exists $c=c(\varepsilon)>0$ such that

$$
\frac{\psi\left(r \sqrt{\lambda} \sqrt{s}^{-1}\right)}{\psi\left(\sqrt{s}^{-1}\right)} \leq c\left((r \sqrt{\lambda})^{\gamma_{1}-\varepsilon}+(r \sqrt{\lambda})^{\gamma_{1}+\varepsilon}\right) \quad \text { for } s \geq 1,0<r \leq \sqrt{s} \sqrt{\lambda}^{-1} \text {. }
$$


Hence, by (3.15) we may use dominated convergence theorem to $I(s)$ and get

$$
\lim _{s \rightarrow \infty} I(s)=\lambda^{\gamma_{1} / 2} \int_{0}^{\infty} e^{-\frac{r^{2}}{4}}\left|\frac{r^{2}}{4}-\frac{d+2}{2}\right| r^{\gamma_{1}+d-1} d r<\infty .
$$

On the other hand, by the change of variable $v=r \sqrt{\lambda} \sqrt{s}^{-1}$ we have that

$$
\begin{aligned}
I I(s) & =\int_{\sqrt{s} \sqrt{\lambda}^{-1}}^{\infty} e^{-\frac{r^{2}}{4}}\left(\frac{\psi\left(r \sqrt{\lambda} \sqrt{s}^{-1}\right)}{\psi\left(\sqrt{s}^{-1}\right)}\right)^{2} r^{d+1} d r \\
& =\psi\left(\sqrt{s}^{-1}\right)^{-2} \int_{1}^{\infty} e^{-\frac{s v^{2}}{4 \lambda}} \psi(v)^{2}\left(\sqrt{s} \sqrt{\lambda}^{-1}\right)^{d+2} v^{d+1} d v
\end{aligned}
$$

By Lemma 3.1, for $s \geq \lambda$

$$
\begin{aligned}
& \int_{1}^{\infty} e^{-\frac{s v^{2}}{4 \lambda}} \psi(v)^{2}\left(\sqrt{s} \sqrt{\lambda}^{-1}\right)^{d+2} v^{d+1} d v \\
\leq & 8\left(\sup _{a \leq 1} \psi(a)^{2}\right) \int_{1}^{\infty} e^{-\frac{s v^{2}}{4 \lambda}}\left(\sqrt{s} \sqrt{\lambda}^{-1}\right)^{d+2} v^{d+5} d v \\
= & 8\left(\sup _{a \leq 1} \psi(a)^{2}\right) s^{-3} \lambda^{3} \int_{1}^{\infty} e^{-\frac{s v^{2}}{4 \lambda}}\left(v \sqrt{s} \sqrt{\lambda}^{-1}\right)^{d+8} v^{-3} d v \\
\leq & 8\left(\sup _{a \leq 1} \psi(a)^{2}\right) s^{-3} \lambda^{3}\left(\sup _{b \geq 1} b^{d+8} e^{-\frac{b^{2}}{4}}\right) \int_{1}^{\infty} v^{-3} d v \\
= & 4\left(\sup _{a \leq 1} \psi(a)^{2}\right) s^{-3} \lambda^{3}\left(\sup _{b \geq 1} b^{d+8} e^{-\frac{b^{2}}{4}}\right) .
\end{aligned}
$$

Thus, since $a \rightarrow \psi\left(\sqrt{a}^{-1}\right)$ varies regularly at $\infty$ with index $\gamma_{1} / 2 \in[0,1]$,

$$
0 \leq \limsup _{s \rightarrow \infty} I I(s) \leq 4 \lambda^{3}\left(\sup _{a \leq 1} \psi(a)^{2}\right)\left(\sup _{b \geq 1} b^{d+6} e^{-\frac{b^{2}}{8}}\right)\left(\limsup _{s \rightarrow \infty} \frac{s^{-3 / 2}}{\psi\left(\sqrt{s}^{-1}\right)}\right)^{2}=0
$$

Therefore we conclude that the integral term in (3.14) goes to 0 if $s \rightarrow \infty$ and $t \frac{\psi\left(\sqrt{s}^{-1}\right)^{2}}{H\left(s^{-1}\right)} \rightarrow 0$. This and regular variation of $H$ with index $\gamma$ (by Proposition 3.4) yield

$$
\lim _{\substack{s \rightarrow \infty \\ t \frac{\psi(\sqrt{s}-1)^{2}}{H\left(s^{-1}\right)} \rightarrow 0}} \int_{0}^{\infty} e^{-\lambda r} \frac{r \mathbb{P}\left(\left|X_{t}\right| \geq \sqrt{r} \sqrt{s}\right)}{t H\left(s^{-1}\right)} d r=\lambda^{\gamma-2}=\int_{0}^{\infty} e^{-\lambda r} \frac{r^{1-\gamma}}{\Gamma(2-\gamma)} d r .
$$

By Theorem 2.1 it follows that

$$
\lim _{\substack{s \rightarrow \infty \\ t \frac{\psi(\sqrt{s}-1)^{2}}{H\left(s^{-1}\right)} \rightarrow 0}} \int_{0}^{R} \frac{r \mathbb{P}\left(\left|X_{t}\right| \geq \sqrt{r} \sqrt{s}\right)}{t H\left(s^{-1}\right)} d r=\int_{0}^{R} \frac{r^{1-\gamma}}{\Gamma(2-\gamma)} d r=\frac{R^{2-\gamma}}{\Gamma(3-\gamma)}
$$

for any $R>0$. 
Proposition 3.6. Assume that $H(\lambda)=\phi(\lambda)-\lambda \phi^{\prime}(\lambda)$ varies regularly at 0 (at $\infty$, respectively) with index $\gamma \in[0,2)$ and $\psi$ varies regularly at 0 (at $\infty$, respectively) with index $\gamma_{1} \in[0,2]$. Then

$$
\mathbb{P}\left(\left|X_{t}\right| \geq r\right) \sim \frac{1}{\Gamma(2-\gamma)} t H\left(r^{-2}\right), \quad r \rightarrow \infty(r \rightarrow 0, \text { resp. }) \text { and } t \frac{\psi\left(r^{-1}\right)^{2}}{H\left(r^{-2}\right)} \rightarrow 0 .
$$

Proof. By Lemma 3.5, we have that for $0<a<b$

$$
\lim _{\substack{r \rightarrow \infty(r \rightarrow 0, \text { resp. }) \\ t \frac{\psi\left(r^{-1}\right)^{2}}{H\left(r^{-1}\right)} \rightarrow 0}} \frac{\int_{a}^{b} s \mathbb{P}\left(\left|X_{t}\right| \geq \sqrt{r s}\right) d s}{t H\left(r^{-1}\right)}=\frac{b^{2-\gamma}-a^{2-\gamma}}{\Gamma(3-\gamma)},
$$

which implies that

$$
\begin{aligned}
\limsup _{\substack{r \rightarrow \infty(r \rightarrow 0, \text { resp. }) \\
t \frac{\psi(\sqrt{r}-1)^{2}}{H\left(r^{-1}\right)} \rightarrow 0}} \frac{b^{2}-a^{2}}{2} \frac{\mathbb{P}\left(\left|X_{t}\right| \geq \sqrt{b r}\right)}{t H\left(r^{-1}\right)} & \leq \frac{b^{2-\gamma}-a^{2-\gamma}}{\Gamma(3-\gamma)} \\
& \leq \limsup _{\substack{r \rightarrow \infty(r \rightarrow 0, \text { resp. }) \\
t \frac{\psi\left(\sqrt{r^{-1}}\right)^{2}}{H\left(r^{-1}\right)} \rightarrow 0}} \frac{b^{2}-a^{2}}{2} \frac{\mathbb{P}\left(\left|X_{t}\right| \geq \sqrt{a r}\right)}{t H\left(r^{-1}\right)} .
\end{aligned}
$$

By taking $b=1>a$ we get

$$
\limsup _{\substack{r \rightarrow \infty(r \rightarrow 0, \text { resp. }) \\ t \frac{\psi(\sqrt{r}-1)^{2}}{H\left(r^{-1}\right)} \rightarrow 0}} \frac{\mathbb{P}\left(\left|X_{t}\right| \geq \sqrt{r}\right)}{t H\left(r^{-1}\right)} \leq \lim _{a \uparrow 1} \frac{1-a^{2-\gamma}}{1-a^{2}} \frac{2}{\Gamma(3-\gamma)}=\frac{1}{\Gamma(2-\gamma)} .
$$

Similarly, for $a=1<b$ we obtain

$$
\liminf _{\substack{r \rightarrow \infty(r \rightarrow 0, \text { resp. }) \\ t \frac{\psi(\sqrt{r}-1)^{2}}{H\left(r^{-1}\right)} \rightarrow 0}} \frac{\mathbb{P}\left(\left|X_{t}\right| \geq \sqrt{r}\right)}{t H\left(r^{-1}\right)} \geq \lim _{b \downarrow 1} \frac{b^{2-\gamma}-1}{b^{2}-1} \frac{2}{\Gamma(3-\gamma)}=\frac{1}{\Gamma(2-\gamma)} .
$$

We now observe the following fact.

Proposition 3.7. Assume that $g(\lambda)=\psi(\lambda)-\frac{\lambda}{2} \psi^{\prime}(\lambda)$ varies regularly at 0 (at $\infty$, respectively) with index $2 \gamma$ with $\gamma \geq 0$. We further assume that, if $g$ varies regularly at $\infty$ with index $2 \gamma \in[0,2)$, then the diffusion part is zero. Then $\psi$ varies regularly at 0 (at $\infty$, respectively) with index $2(1 \wedge \gamma)$.

Proof. Since

$$
\left(\log \frac{\psi(t)}{t^{2}}\right)^{\prime}=\frac{\psi^{\prime}(t)}{\psi(t)}-\frac{2}{t}=-\frac{2 g(t)}{t \psi(t)}
$$


by integrating it we obtain

$$
\log \frac{\psi(\lambda)}{\lambda^{2}}=\log \psi(1)+\int_{\lambda}^{1} \frac{2 g(t)}{t \psi(t)} d t .
$$

Hence,

$$
\frac{\psi(\lambda)}{\lambda^{2}}=\psi(1) \exp \left(\int_{\lambda}^{1} \frac{2 g(t)}{\psi(t)} \frac{d t}{t}\right) \quad \text { for } \lambda>0
$$

On the other hand, since $\left(\frac{\psi(\lambda)}{\lambda^{2}}\right)^{\prime}=-2 \lambda^{-3} g(\lambda)$, we have

$$
\frac{\psi(\lambda)}{\lambda^{2}}-\frac{\psi(R)}{R^{2}}=\int_{\lambda}^{R} \frac{2 g(s)}{s^{3}} d s
$$

Thus for $\lambda>0$ and $\theta \geq 1$

$$
\frac{\psi(\lambda)}{g(\lambda)}=\frac{\psi(\lambda) / \lambda^{2}}{g(\lambda) / \lambda^{2}}=\int_{\lambda}^{\theta \lambda} \frac{2 \lambda^{2} g(s)}{s^{3} g(\lambda)} d s+\frac{\psi(\theta \lambda)}{\theta^{2} g(\lambda)}=\int_{1}^{\theta} \frac{2 g(\lambda s)}{s^{3} g(\lambda)} d s+\frac{\psi(\theta \lambda)}{\theta^{2} g(\lambda)} .
$$

(1) We first assume $g$ varies regularly at 0 with index $2 \gamma$ with $\gamma \in[0,1)$. Then, by Potter's theorem (see [BGT87, Theorem 1.5.6]), there exists a constant $c_{1}>0$ such that

$$
\frac{g(\lambda s)}{s^{3} g(\lambda)} \leq c_{1} s^{\gamma-2} \quad \text { for } 0<\lambda<\lambda s<1 \text { and } s>1
$$

Thus by the dominated convergence theorem, from (3.18) we have

$$
\lim _{\lambda \rightarrow 0} \frac{\psi(\lambda)}{g(\lambda)}=\lim _{\lambda \rightarrow 0}\left(\int_{1}^{1 / \lambda} \frac{2 g(\lambda s)}{s^{3} g(\lambda)} d s+\psi(1) \frac{\lambda^{2}}{g(\lambda)}\right)=\int_{1}^{\infty} 2 s^{2 \gamma-3} d s=\frac{1}{1-\gamma}
$$

Therefore $\lim _{\lambda \rightarrow 0} \frac{2 g(\lambda)}{\psi(\lambda)}=2-2 \gamma$. Now, using the zero version of [BGT87, Theorem 1.3.1 and (1.5.2)] we conclude from (3.16) and the above display that $\psi(\lambda) / \lambda^{2}$ varies regularly at 0 with index $2 \gamma-2$. Therefore, $\psi$ varies regularly at 0 with index $2 \gamma$.

(2) Assume that $g$ varies regularly at $\infty$ with index $2 \gamma$ with $\gamma \in[0,1)$ and the diffusion part is zero. Then

$$
\lim _{\lambda \rightarrow \infty} \frac{\psi(\lambda)}{\lambda^{2}}=0
$$

Hence, by letting $R \rightarrow \infty$ in (3.17) we obtain

$$
\frac{\psi(\lambda)}{\lambda^{2}}=\int_{\lambda}^{\infty} \frac{2 g(s)}{s^{2}} \frac{d s}{s} .
$$

(3.19) and [BGT87, Proposition1.5.10] imply that $\psi$ also varies regularly at $\infty$ with index $2 \gamma$ with $\gamma \in[0,1)$. 
(3) Assume that $g$ varies regularly at 0 (at $\infty$, respectively) with index $2 \gamma \geq 2$. Proposition 3.4 implies that the term $\frac{\psi(\theta \lambda)}{\theta^{2} g(\lambda)}$ in (3.18) stays positive for small $\lambda$ (large $\lambda$, respectively). Thus, from (3.18), we have that for any $\theta \geq 1$

$$
\liminf _{\substack{\lambda \downarrow 0 \\(\lambda \uparrow \infty, \text { resp. })}} \frac{\psi(\lambda)}{g(\lambda)} \geq 2 \int_{1}^{\theta} s^{2 \gamma-3} d s= \begin{cases}2 \log \theta & \text { if } \gamma=1 \\ \frac{1}{\gamma-1}\left(\theta^{2(\gamma-1)}-1\right) & \text { if } \gamma>1\end{cases}
$$

where we have used the fact

$$
\lim _{\substack{\lambda \downarrow 0 \\(\lambda \uparrow \infty, \text { resp. })}} \frac{g(\lambda s)}{g(\lambda)}=s^{2 \gamma} \quad \text { uniformly in } s \in[1, \theta] .
$$

(See [BGT87, Theorem 1.5.2].) Therefore, by letting $\theta \rightarrow \infty$, we get

$$
\lim _{\substack{t \downarrow 0 \\(t \uparrow \infty, \text { resp. })}} \frac{g(t)}{\psi(t)}=\left(\lim _{\substack{t \downarrow 0 \\ t \uparrow \infty, \text { resp. })}} \frac{\psi(t)}{g(t)}\right)^{-1}=0 .
$$

Using [BGT87, Theorem 1.3.1] we conclude from (3.16) and (3.20) that $\frac{\psi(\lambda)}{\lambda^{2}}$ varies slowly at 0 (at $\infty$, respectively). Therefore, $\psi$ varies regularly at 0 (at $\infty$, respectively) with index 2 .

The next corollary follows immediately from (3.20).

Corollary 3.8. If $g$ varies regularly at $\infty$ with index $\alpha \geq 0$. Then, the index $\alpha$ must be in $[0,2]$.

Proof of Theorem 1.3. (i) By Proposition 3.7 we have that $\psi$ varies regularly at 0 with index $2 \wedge \alpha$. Moreover, by Proposition 3.4 we have that $H$ varies regularly at 0 with index $\alpha / 2$. Noting that

$$
\frac{\psi\left(r^{-1}\right)}{1-\frac{r^{-1} \psi^{\prime}\left(r^{-1}\right)}{2 \psi\left(r^{-1}\right)}}=\frac{\psi\left(r^{-1}\right)^{2}}{H\left(r^{-2}\right)} \frac{H\left(r^{-2}\right)}{g\left(r^{-1}\right)}
$$

we now use Propositions 3.4 and 3.6 to get

$$
\begin{aligned}
\lim _{r \rightarrow \infty, \frac{t \psi\left(r^{-1}\right)}{1-\frac{r^{-1} \psi^{\prime}\left(r^{-1}\right)}{2 \psi\left(r^{-1}\right)}} \rightarrow 0} \frac{\mathbb{P}\left(\left|X_{t}\right| \geq r\right)}{t g\left(r^{-1}\right)} & =\lim _{r \rightarrow \infty, \frac{t \psi\left(r^{-1}\right)}{1-\frac{r^{-1} \psi^{\prime}\left(r^{-1}\right)}{2 \psi\left(r^{-1}\right)}}} \frac{\mathbb{P}\left(\left|X_{t}\right| \geq r\right)}{t H\left(r^{-2}\right)} \frac{H\left(r^{-2}\right)}{g\left(r^{-1}\right)} \\
& =\frac{1}{\Gamma\left(2-\frac{\alpha}{2}\right)} \frac{2^{\alpha-1} \Gamma\left(\frac{d+\alpha}{2}\right)}{\Gamma\left(\frac{d}{2}\right)} .
\end{aligned}
$$

(ii) The first claim in (ii) is Corollary 3.8. The proof of the second claim in (ii) is analogous to the proof of (i). 
Remark 3.9. If $\psi$ varies regularly at $\infty$, with index $\alpha \in[0,2)$ then it follows from Proposition 3.3 that $H$ varies regularly with index $\alpha / 2$. Thus Propositions 3.3 and 3.6 yield

$$
\begin{aligned}
\lim _{\substack{r \rightarrow \infty(r \rightarrow 0, \text { resp. }) \\
t \psi\left(r^{-1}\right) \rightarrow 0}} \frac{\mathbb{P}\left(\left|X_{t}\right| \geq r\right)}{t \psi\left(r^{-1}\right)} & =\lim _{\substack{r \rightarrow \infty(r \rightarrow 0, \text { resp. }) \\
t \frac{\psi\left(r^{-1}\right)^{2}}{H\left(r^{-2}\right)} \rightarrow 0}} \frac{\mathbb{P}\left(\left|X_{t}\right| \geq r\right)}{t H\left(r^{-2}\right)} \frac{H\left(r^{-2}\right)}{\psi\left(r^{-1}\right)} \\
& =\frac{1}{\Gamma\left(2-\frac{\alpha}{2}\right)} \frac{(2-\alpha) 2^{\alpha-1} \Gamma\left(\frac{d+\alpha}{2}\right)}{\Gamma\left(\frac{d}{2}\right)} .
\end{aligned}
$$

\section{Asymptotic of the transition Density of unimodal LÉvy PROCEsses}

Throughout this section we assume that $X$ is a unimodal Lévy process in $\mathbb{R}^{d}, d \geq 1$ with the characteristic exponent $\xi \rightarrow \psi(|\xi|)$, and that $p(t, x)$ and $J(x)$ are the transition density and the jumping kernel of $X_{t}$, respectively.

We will give the precise off-diagonal asymptotic expression of $p(t, x)$. Recall that $p(t, x)=q(t,|x|)$ where $r \rightarrow q(t, r)$ is decreasing. We also recall that $\phi:(0, \infty) \rightarrow$ $(0, \infty)$ is defined in $(3.4)$.

Theorem 4.1. Assume that $H(\lambda)=\phi(\lambda)-\lambda \phi^{\prime}(\lambda)$ varies regularly at 0 (at $\infty$, respectively) with index $\gamma \in(0,2)$ and $\psi$ varies regularly at 0 (at $\infty$, respectively) with index $\gamma_{1} \in[0,2]$. Then

$$
\begin{gathered}
p(t, x) \sim \frac{\gamma \pi^{-d / 2} \Gamma\left(\frac{d}{2}\right)}{\Gamma(2-\gamma)} t|x|^{-d} H\left(|x|^{-2}\right), \quad|x| \rightarrow \infty(x \rightarrow 0, \text { resp. }) \\
\text { and } t \frac{\psi\left(|x|^{-1}\right)^{2}}{H\left(|x|^{-2}\right)} \rightarrow 0 .
\end{gathered}
$$

Proof. Let $0<a<b$. By Proposition 3.6 we have

$$
\begin{aligned}
& \lim _{\substack{r \rightarrow \infty(r \rightarrow 0, \text { resp. }) \\
t \frac{\psi(\sqrt{r}-1)^{2}}{H\left(r^{-1}\right)} \rightarrow 0}} \frac{\mathbb{P}\left(b^{-1} r \leq\left|X_{t}\right|<a^{-1} r\right)}{t H\left(r^{-2}\right)} \\
= & \lim _{\substack{r \rightarrow \infty(r \rightarrow 0, \text { resp. }) \\
t \frac{\psi(\sqrt{r}-1)^{2}}{H\left(r^{-1}\right)} \rightarrow 0}}\left(\frac{\mathbb{P}\left(\left|X_{t}\right| \geq b^{-1} r\right)}{t H\left(r^{-2}\right)}-\frac{\mathbb{P}\left(\left|X_{t}\right| \geq a^{-1} r\right)}{t H\left(r^{-2}\right)}\right)=\frac{b^{2 \gamma}-a^{2 \gamma}}{\Gamma(2-\gamma)} .
\end{aligned}
$$

Hence, by using

$$
\mathbb{P}\left(\left|X_{t}\right| \geq r\right)=c_{d} \int_{r}^{\infty} q(t, s) s^{d-1} d s
$$


where $c_{d}=\frac{2 \pi^{d / 2}}{\Gamma(d / 2)}$, and the fact that $p(t, x)$ is radially decreasing, for $a<1<b$ we obtain

$$
\begin{aligned}
& \limsup _{\substack{|x| \rightarrow \infty(|x| \rightarrow 0, \text { resp. }) \\
t \frac{\psi\left(|x|^{-1}\right)^{2}}{H\left(|x|^{-2}\right)} \rightarrow 0}} \frac{p(t, x) c_{d} d^{-1}\left(1-b^{-d}\right)}{t|x|^{-d} H\left(|x|^{-2}\right)} \\
\leq & \limsup _{\substack{r \rightarrow \infty(r \rightarrow 0, \text { resp. }) \\
t \frac{\psi\left(r^{-1}\right)^{2}}{H\left(r^{-2}\right)} \rightarrow 0}} \frac{\mathbb{P}\left(\left|X_{t}\right| \geq b^{-1} r\right)-\mathbb{P}\left(\left|X_{t}\right| \geq r\right)}{t H\left(r^{-2}\right)} \leq \frac{b^{2 \gamma}-1}{\Gamma(2-\gamma)}
\end{aligned}
$$

and

$$
\begin{aligned}
& \liminf _{\substack{|x| \rightarrow \infty(|x| \rightarrow 0, \text { resp. }) \\
t \frac{\psi\left(|x|^{-1}\right)^{2}}{H\left(|x|^{-2}\right)} \rightarrow 0}} \frac{p(t, x) c_{d} d^{-1}\left(a^{-d}-1\right)}{t|x|^{-d} H\left(|x|^{-2}\right)} \\
\geq & \liminf _{\substack{r \rightarrow \infty(r \rightarrow 0, \text { resp. }) \\
t \frac{\psi\left(r^{-1}\right)^{2}}{H\left(r^{-2}\right)} \rightarrow 0}} \frac{\mathbb{P}\left(\left|X_{t}\right| \geq r\right)-\mathbb{P}\left(\left|X_{t}\right| \geq a^{-1} r\right)}{t H\left(r^{-2}\right)} \geq \frac{a^{2 \gamma}-1}{\Gamma(2-\gamma)} .
\end{aligned}
$$

Note that in the case $\gamma=0$ formula holds.

If $\gamma>0$, we let $a \uparrow 1$ and $b \downarrow 1$ to obtain

$$
\lim _{\substack{|x| \rightarrow \infty(|x| \rightarrow 0, \text { resp. }) \\ t \frac{\psi\left(|x|^{-1}\right)^{2} \rightarrow 0}{H\left(|x|^{-2}\right)}}} \frac{p(t, x)}{t|x|^{-d} H\left(|x|^{-2}\right)}=\frac{\gamma \pi^{-d / 2} \Gamma\left(\frac{d}{2}\right)}{\Gamma(2-\gamma)} .
$$

By a consequence of Theorem 4.1 we prove Theorem 1.4 which cover [CGT15, Theorems 4 and 5] and we further obtain a new result for $\alpha=2$.

Proof of Theorem 1.4. By Propositions 3.7 and 3.4 we have that $\psi$ varies regularly at 0 (at $\infty$, respectively) with index $2 \wedge \alpha$. and $H$ varies regularly at 0 (at $\infty$, respectively) with index $\alpha / 2$. By (3.21), (1.6) now follows immediately from Theorem 4.1 and Proposition 3.4 to get

$$
\begin{aligned}
\lim _{\substack{|x| \rightarrow \infty(|x| \rightarrow 0, \text { resp. }) \\
\frac{t \psi\left(|x|^{-1}\right)}{1-\frac{|x|^{-1} \psi^{\prime}\left(\left.|x|\right|^{-1}\right)}{2 \psi\left(|x|^{-1}\right)}} \rightarrow 0}} \frac{p(t, x)}{t|x|^{-d} g\left(|x|^{-1}\right)} & =\frac{\alpha \pi^{-d / 2} \Gamma\left(\frac{d}{2}\right)}{\Gamma\left(2-\frac{\alpha}{2}\right)} \frac{2^{\alpha-1} \Gamma\left(\frac{d+\alpha}{2}\right)}{\Gamma\left(\frac{d}{2}\right)} \\
& =\alpha 2^{\alpha-1} \pi^{-d / 2} \frac{\Gamma\left(\frac{d+\alpha}{2}\right)}{\Gamma\left(2-\frac{\alpha}{2}\right)} .
\end{aligned}
$$


(1.7) follows from (1.6) and the fact that $\lim _{t \downarrow 0} t^{-1} p(t, x)=J(x)$ vaguely on $\mathbb{R}^{d} \backslash\{0\}$ (see [CGT15, proof of Theorem 6] too).

Remark 4.2. (i) From the proof of Theorem 1.4, we see that if $g$ varies slowly at 0 (at $\infty$, respectively) and $\psi$ does not have diffusion part then

$$
\lim _{\substack{|x| \rightarrow \infty(|x| \rightarrow 0, \text { resp. }) \\ \frac{t \psi\left(\left.|x|\right|^{-1}\right)}{1-\frac{|x|^{-1} \psi^{\prime}\left(|x|^{-1}\right)}{2 \psi\left(|x|^{-1}\right)}} \rightarrow 0}} \frac{p(t, x)}{t|x|^{-d} g\left(|x|^{-1}\right)}=0 .
$$

(ii) If $\psi$ varies regularly at 0 (at $\infty$, respectively), with index $\alpha \in[0,2)$ then it follows from Proposition 3.3 that $H$ varies regularly at 0 (at $\infty$, respectively) with index $\alpha / 2$. Thus, Theorem 4.1 and Proposition 3.3 yield

$$
\begin{aligned}
\lim _{\substack{|x| \rightarrow \infty\left(|x| \rightarrow 0, \text { resp.) } \\
t \psi\left(|x|^{-1}\right) \rightarrow 0\right.}} \frac{p(t, x)}{t|x|^{-d} \psi\left(|x|^{-1}\right)} & =\lim _{\substack{|x| \rightarrow \infty\left(|x|^{-\rightarrow 0, \text { resp. }} \\
t \frac{\psi\left(|x|^{-1}\right)^{2}}{H\left(|x|^{-2}\right)} \rightarrow 0\right.}} \frac{p(t, x)}{t|x|^{-d} H\left(|x|^{-2}\right)} \frac{H\left(|x|^{-2}\right)}{\psi\left(|x|^{-1}\right)} \\
& =\frac{2^{-1} \alpha \pi^{-d / 2} \Gamma\left(\frac{d}{2}\right)}{\Gamma\left(2-\frac{\alpha}{2}\right)} \frac{(2-\alpha) 2^{\alpha-1} \Gamma\left(\frac{d+\alpha}{2}\right)}{\Gamma\left(\frac{d}{2}\right)} \\
& =\alpha 2^{\alpha-1} \pi^{-d / 2} \frac{\Gamma\left(\frac{d+\alpha}{2}\right)}{\Gamma\left(1-\frac{\alpha}{2}\right)} .
\end{aligned}
$$

We apply Theorem 1.4 to subordinate Brownian motion $X=\left(X_{t}\right)_{t \geq 0}$. It is an isotropic Lévy process in $\mathbb{R}^{d}(d \geq 1)$ defined by

$$
X_{t}=B_{T_{t}}, \quad t \geq 0,
$$

where $B=\left(B_{t}\right)_{t \geq 0}$ is the $d$-dimensional Brownian motion with the transition density

$$
p^{(2)}(t, x)=(4 \pi t)^{-d / 2} e^{-\frac{|x|^{2}}{4 t}}, \quad t>0, x \in \mathbb{R}^{d} .
$$

and $T=\left(T_{t}\right)_{t \geq 0}$ is an independent subordinator whose the Laplace exponent is $\varphi$.

It is known that the characteristic exponent of $X$ is given by $\psi(\xi)=\varphi\left(|\xi|^{2}\right)$ and $X$ has a transition transition density given by

$$
p(t, x)=\int_{(0, \infty)}(4 \pi s)^{-d / 2} e^{-\frac{|x|^{2}}{4 s}} \mathbb{P}\left(T_{t} \in d s\right) .
$$

Thus, subordinate Brownian motion is a subclass of unimodal Lévy processes and the next corollary follows directly from Theorem 1.4.

Corollary 4.3. $X_{t}=B_{T_{t}}$ is a subordinate Brownian motion and $\varphi$ is the Laplace exponent of the subordinator $T_{t}$. Suppose $H(\lambda)=\varphi(\lambda)-\lambda \varphi^{\prime}(\lambda)$ varies regularly at 0 
(at $\infty$, respectively) with index $\gamma \in(0,2)$. We further assume that $\varphi$ does not have drift part if $H$ varies regularly at $\infty$ with index $\gamma \in(0,1)$. Then

$$
\begin{aligned}
p(t, x) \sim \gamma 4^{\gamma} \pi^{-d / 2} \frac{\Gamma\left(\frac{d}{2}+\gamma\right)}{\Gamma(2-\gamma)} t|x|^{-d} H\left(|x|^{-2}\right) \\
|x| \rightarrow \infty(x \rightarrow 0, \text { resp. }) \quad \text { and } \quad \frac{t \varphi\left(|x|^{-2}\right)^{2}}{H\left(|x|^{-2}\right)} \rightarrow 0 .
\end{aligned}
$$

Example 4.4. Let us consider a Bernstein function $\varphi(\lambda)=\lambda \log \left(1+\frac{1}{\lambda}\right)$. (see table entry No. 27 on page 316 in [SSV12]). Then $\varphi$ varies regularly at 0 with index 1 and we have $H(\lambda)=\lambda /(\lambda+1)$ so that

$$
\lim _{\lambda \downarrow 0} \frac{H(\lambda)}{\lambda}=1 \quad \text { and } \quad \frac{\varphi(\lambda)}{H(\lambda)} \sim \log \left(1+\frac{1}{\lambda}\right), \quad \lambda \downarrow 0 .
$$

By (4.2) we have the asymptotic formula as

$$
\lim _{\substack{|x| \rightarrow \infty \\ t|x|^{-2} \log (1+|x|)^{2} \rightarrow 0}} \frac{p(t, x)}{t|x|^{-d-2}}=4 \pi^{-d / 2} \Gamma\left(\frac{d+2}{2}\right) .
$$

Acknowledgements: The first named author is grateful to Joohak Bae for reading the earlier draft of this paper and giving helpful comments. We thank the referees for helpful comments on the first version of this paper.

\section{REFERENCES}

[Ben94] A. Bendikov, Asymptotic formulas for symmetric stable semigroups, Exposition. Math. 12 (1994), no. 4, 381-384. MR 1297844

[BG60] R. M. Blumenthal and R. K. Getoor, Some theorems on stable processes, Trans. Amer. Math. Soc. 95 (1960), 263-273.

[BGT87] N. H. Bingham, C. M. Goldie, and J. L. Teugels, Regular variation, Cambridge University Press, Cambridge, 1987.

[CGT15] W. Cygan, T. Grzywny, and B. Trojan, Asymptotic behavior of densities of unimodal convolution semigroups, to appear in Trans. Amer. Math. Soc. (2015).

[Fel71] W. Feller, An introduction to probability theory and its applications, John Wiley and Sons, New York, 1971.

[FLH09] J. E. Figueroa-López and C. Houdré, Small-time expansions for the transition distributions of Lévy processes, Stochastic Process. Appl. 119 (2009), no. 11, 3862-3889. MR 2552308

[Grz14] T. Grzywny, On Harnack inequality and Hölder regularity for isotropic unimodal Lévy processes, Potential Anal. 41 (2014), 1-29.

[Ish94] Y. Ishikawa, Asymptotic behavior of the transition density for jump type processes in small time, Tohoku Math. J. (2) 46 (1994), no. 4, 443-456. MR 1301283

[JP87] N. C. Jain and W. E. Pruitt, Lower tail probability estimates for subordinators and nondecreasing random walks, Ann. Probab. 15(1) (1987), 75-101. 
[Kno11] V. Knopova, Asymptotic behaviour of the distribution density of some Lévy functionals in $\mathbb{R}^{n}$, Theory Stoch. Process. 17 (2011), no. 2, 35-54. MR 2934558

[Léa87] R. Léandre, Densité en temps petit d'un processus de sauts, Séminaire de Probabilités, XXI, Lecture Notes in Math., vol. 1247, Springer, Berlin, 1987, pp. 81-99. MR 941977

[Mim] A. Mimica, Heat kernel estimates for subordinate brownian motions, to appear in Proc. Lond. Math. Soc., arXiv:1503.01600, 2015.

[SSV12] R. L. Schilling, R. Song, and Z. Vondraček, Bernstein functions, second ed., de Gruyter Studies in Mathematics, vol. 37, Walter de Gruyter \& Co., Berlin, 2012, Theory and applications. MR 2978140

Department of Mathematical Sciences, Seoul National University, Building 27, 1 Gwanak-ro, Gwanak-gu Seoul 08826, Republic of Korea

E-mail address: pkim@snu.ac.kr

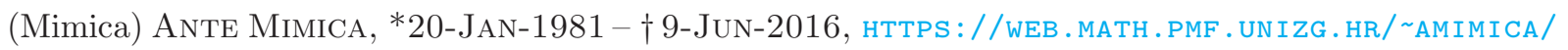

